\title{
Flora de la costa de OAXACA, MÉXico (2): LISTA FLORÍSTICA COMENTADA DEL Parque Nacional huatulco
}

\author{
Silvia H. Salas-Morales', Leo Schibli ${ }^{\dagger}$, Arturo Nava-Zafra y Alfredo Saynes-Vásquez \\ Sociedad para el Estudio de los Recursos Bióticos de Oaxaca (SERBO, A.C.). \\ Camino Nacional No. 80, San Sebastián Tutla, 71246, Oaxaca, México. \\ 'Autor para la correspondencia. Correo-e: serbo@prodigy.net.mx
}

\begin{abstract}
Resumen: Se presenta la lista de especies, incluyendo algunas de sus características, así como la descripción florística de los tipos de vegetación del Parque Nacional Huatulco. El estudio consistió en 18 recorridos de campo durante los cuales se recolectaron 4,382 números de plantas vasculares, en altitudes entre 0 y $200 \mathrm{~m}$. De estas colecciones se han determinado 3,312 muestras y hasta el momento se tienen 91 familias, 391 géneros y 736 especies. Las familias dominantes por su riqueza de especies son Leguminosae (146), Euphorbiaceae (48), Asteraceae (42) y Convolvulaceae (37). Los tipos de vegetación reconocidos en las 6,374.98 ha del Parque son: bosque de galería, manglar, sabana, selva baja caducifolia, selva baja espinosa, selva mediana subcaducifolia, vegetación de dunas costeras y vegetación secundaria.
\end{abstract}

Palabras clave: costa del Pacífico, flora, Huatulco, trópico estacionalmente seco, vegetación.

\begin{abstract}
An annotated list of vascular plant species and a floristic description of the vegetation types of the Huatulco National Park, Oaxaca, Mexico, are presented. Floristic exploration upon which the list is based took place in an area of approximately 6,374.98 ha. The study consisted of 18 collecting trips during which 4,382 numbers of vascular plants were collected, at altitudes ranging from sea level to $200 \mathrm{~m}$. Of the 3,312 collections determined so far, 91 families, 391 genera, and 736 species are reported. The dominant families in number of species are Leguminosae (146), Euphorbiaceae (48), Asteraceae (42), and Convolvulaceae (37). Vegetation types recognized are coastal dune vegetation, gallery forest, mangroves, thorn forest, tropical deciduous forest, tropical subdeciduous forest, savanna, and secondary vegetation.
\end{abstract}

Keywords: flora, Huatulco, Pacific coast, seasonally dry tropics, tropical dry forest, vegetation.

$\mathbf{M}$ ucho se ha dicho sobre la diversidad florística de la República Mexicana y particularmente del estado de Oaxaca. Aunque no se conoce con exactitud el número de especies vegetales que habitan en el estado, se han hecho diferentes estimaciones, entre las que cabe mencionar las de Toledo (1988), Lorence y García-Mendoza (1989), Rzedowski (1991) y más recientemente García-Mendoza (2004). Este último reportó 8,431 especies de plantas vasculares

A pesar de los esfuerzos realizados hasta el momento, existen todavía áreas del estado que se desconocen, como la Sierra Sur, para las que existe un gran vacío de información florística. García-Mendoza y Torres-Colín (1999) afirmaron que una de las regiones fisiográfico-florísticas menos exploradas es la Planicie Costera del Pacífico. Sin embargo, el personal de la Sociedad para el Estudio de los Recursos Bióticos de Oaxaca (SERBO, A.C.), ha explorado esta región desde hace mas de 10 años y han recolectado en ella numerosos ejemplares botánicos, por lo que actualmente es una de las mejor conocidas, no sólo en Oaxaca, sino en toda costa del Pacífico en territorio mexicano.

La vegetación dominante de esta región biogeográfica es la selva baja caducifolia, que es una comunidad vegetal ampliamente distribuida en el estado (Torres-Colín, 2004). Lott y Atkinson (2006) incluyen a esta región de la costa de Oaxaca en lo que denominan subregión Costa Central, la cual contiene las extensiones de selva seca más amplias y mejor conservadas (Salas-Morales et al., 2003); junto con Caleta de Campo, Michoacán, aquí se localiza uno de los 
sitios con mayor diversidad de especies en áreas de 0.1 ha (Trejo, 1998).

El Pa rque Nacional Huatulco se encuentra en esta región, propuesta en 1991 como prioritaria para la conservación de la biodive rsidad (García et al., 1992; Salas-Morales et al., 2003), p ropuesta reconocida por la Comisión Nacional para el Conocimiento y Uso de la Biodive rsidad (CONABIO), al incluir en las áreas de prioridad de conservación a la Sierra Sur y la Costa (Arri aga et al., 2000).

Existen listados florísticos en zonas adyacentes al Parque, como los de González y Miranda (1994) y Escalona (1996), aunque el estudio más completo es el de Salas-Morales et al. (2003). Castillo-Campos et al. (1997) incluyen al Parque en un estudio realizado para el municipio de Santa María Huatulco, en el que presentan una lista florística con 413 especies, 78 familias y 272 géneros. Asimismo, se han realizado estudios sobre la estructura y la diversidad de la vegetación (Salas-Morales, 2002; Trejo y Dirzo, 2002; Gordon et al., 2003, 2004). A pesar del trabajo realizado, es necesario continuar recolectando especímenes en áreas poco conocidas florísticamente, como son los distritos de Jamiltepec y Juquila, y sistematizar la información que ya existe, pues los procesos de deterioro de la selva seca, aunque no han sido evaluados, son alarmantes.

\section{Área de estudio}

El Parque Nacional Huatulco (PNH) se encuentra dentro del área del Desarrollo Turístico Bahías de Huatulco, en la costa oaxaqueña (México). Sus coordenadas extremas son

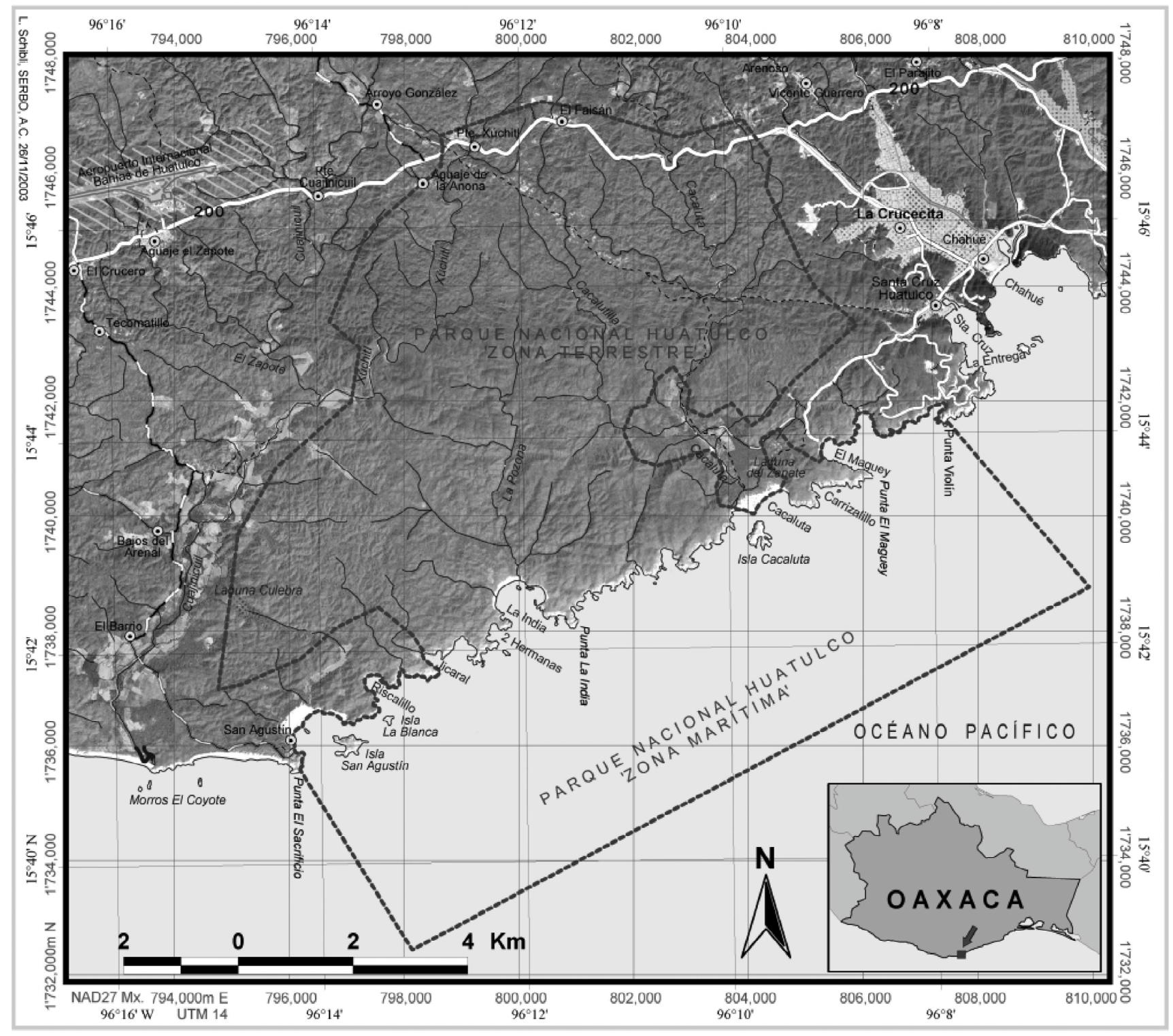

Figura 1. Localización del Parque Nacional Huatulco en la costa de Oaxaca, México 
$15^{\circ} 39^{\prime} 12^{\prime \prime}$ y $15^{\circ} 47^{\prime} 10^{\prime \prime}$ de latitud norte y $96^{\circ} 06^{\prime} 30^{\prime \prime}$ y $96^{\circ} 15^{\prime} 00^{\prime \prime}$ de longitud oeste. Pertenece al municipio de Santa María Huatulco, distrito de Pochutla. La poligonal del PNH delimita un área total de 11,890.98 ha, de las cuales $6,374.98$ son terrestres y 5,516.00 son marinas (CONANP, 2003; figura 1). La zona pertenece a la provincia fisiográfica Sierra Madre del Sur y a la subprovincia Planicie Costera del Pacífico; sus altitudes no rebasan la cota de 200 m s.n.m. (Ferrusquía-Villafranca, 1998). Las rocas predominantes son gneises de edad jurásica, asociados a esquistos, granodiorita gneísica, granulita y metagranito, los cuales forman parte del complejo Xolapa; hay escasos afloramientos de gneises del Precámbrico (INEGI, 1988a). Los suelos dominantes son los Cambisoles crómicos, aunque también se encuentran Litosoles, Regosoles y Feozems háplicos, además de suelos aluviales más profundos (INEGI, 1989).

La región forma parte de la región hidrológica 21 y comprende una serie de cuencas pequeñas independientes constituidas por redes de drenaje dendríticos y subdendríticos (INEGI, 1985). Los ríos que cruzan la zona son el Cuajinicuil-Xúchilt-Arenal, el Todos Santos, el Cacaluta y el Tangolunda; estos escurrimientos forman lagunas intermitentes entre las que destacan La Culebra, La Poza y Cacaluta.

De las nueve bahías principales que integran a las Bahías de Huatulco, cinco quedan comprendidas en el PNH: San Agustín, Chachacual, Cacaluta, Maguey y Órgano, todas con profundidades de menos de $200 \mathrm{~m}$. La costa es afectada por ciclones tropicales cuyas trayectorias impactan directamente al PNH, lo cual convierte a la región en zona de riesgo (CONANP, 2003).

La carencia de estaciones meteorológicas cercanas hace que el clima solamente pueda ser inferido a partir de la cartografía existente. Según ésta, el clima es cálido subhúmedo con lluvias en verano, cuya fórmula climática es Aw'o(w)ig (de acuerdo con el sistema de Köppen modificado por García, 2004). Éste es el clima más seco de los cálidos subhúmedos (CETENAL-UNAM, 1970; INEGI, 1980), aunque la colindancia con el mar tiene una influencia directa sobre la humedad del continente, que es de $37 \%$ (Morales, 1998).

\section{Métodos}

En agosto de 2004 se iniciaron los trabajos para este estudio con la revisión de la carta topográfica de INEGI (1988b), con la finalidad de tener una imagen integral de la zona. Con esta base se programaron los recorridos para la recolecta botánica, el reconocimiento de senderos y las localidades habitadas, y de ubicaron sitios de importancia biológica potencial, como humedales, arroyos y ríos. El material botánico se procesó básicamente en el herbario de SERBO (SERO) y en el Herbario Nacional de la Unive rsidad Nacional Autónoma de México (MEXU). Todos los ejemplares fueron georeferenciados, lo cual permite su ubicación en la cartografía digital disponible. La vegetación se caracterizó con base en la clasificación propuesta por Miranda y Hernández-X. (1963) y Lorence y García-Mendoza (1989).

El sistema de clasificación para las Pteridophyta se basó en Mickel y Beitel (1988) y el de las Magnoliophyta en Cronquist (1981). Se mantuvo el nombre Leguminosae por cuestiones prácticas. Todos los nombres de las plantas y los autores fueron verificados en la base de datos de $\mathrm{W}^{3} \mathrm{TRO}-$ PICOS (mobot.mobot.org/W3T/Search/vast.html). Para cada especie se cuenta con la siguiente información: (1) nombre científico con la autoridad correspondiente, (2) hábito e intervalo de altura de la especie - solamente los reportados en la localidad-, (3) color de la flor o detalles conspicuos de la inflorescencia, siempre y cuando fue posible, (4) mes o meses de floración y/o de fructificación, siempre y cuando fue posible, (5) tipos de vegetación en los que se recolectó, y (6) colector(a) y sus números de colecta.

\section{Resultados}

Flora. El estudio consistió en 18 recorridos de campo, durante los cuales se colectaron 4,382 números de plantas vasculares, con un promedio de cuatro duplicados cada uno, en altitudes que van de 0 a $200 \mathrm{~m}$; de éstos se han determinado 3,312 (76\%). Del material determinado hasta el momento se tienen 91 familias, 391 géneros y 736 especies (cuadro 1; apéndice 1).

Las familias con más especies fueron Leguminosae con

Cuadro 1. Datos sintéticos de la riqueza florística del Parque Nacional Huatulco, Oaxaca, México.

\begin{tabular}{lcccc}
\hline Grupo & Familias & Géneros & Especies & $\begin{array}{c}\text { Categorías } \\
\text { infraespecíficas }\end{array}$ \\
\hline Pteridophyta & $\mathbf{4}$ & $\mathbf{4}$ & $\mathbf{4}$ & - \\
Magnoliophyta & $\mathbf{8 7}$ & $\mathbf{3 8 7}$ & $\mathbf{7 3 2}$ & $\mathbf{2 9}$ \\
$\quad 11$ & 29 & 44 & \\
$\quad$ Monocotiledoneas & 76 & 358 & 688 & $\mathbf{2 9}$ \\
Dicotiledoneas & $\mathbf{9 1}$ & $\mathbf{3 9 1}$ & $\mathbf{7 3 6}$ & $\mathbf{2 9}$ \\
\hline
\end{tabular}


Cuadro 2. Familias con mayor número de especies del Parque Nacional Huatulco, Oaxaca. México.

\begin{tabular}{lccc}
\hline Familia & Número de géneros & $\begin{array}{c}\text { Número de especies } \\
\text { Proporción del total } \\
\text { de especies }(\%)\end{array}$ \\
\hline Leguminosae & 57 & 146 & 19.8 \\
Euphorbiaceae & 18 & 48 & 6.6 \\
Asteraceae & 27 & 42 & 5.8 \\
Convolvulaceae & 6 & 37 & 5.1 \\
Rubiaceae & 17 & 29 & 4 \\
Boraginaceae & 4 & 20 & 2.7 \\
Acanthaceae & 12 & 18 & 2.5 \\
Malvaceae & 11 & 18 & 2.5 \\
Cucurbitaceae & 16 & 17 & 2.3 \\
Bignoniaceae & 12 & 16 & 2.2 \\
Malpighiaceae & 8 & 16 & 2.2 \\
Subtotal & 188 & 407 & 55.3 \\
Otras familias & 203 & 329 & $\mathbf{1 0}$ \\
Total & $\mathbf{3 9 1}$ & $\mathbf{7 3 6}$ & \\
\hline
\end{tabular}

146, Euphorbiaceae con 48, Asteraceae con 42, Convolvulaceae con 37 y Rubiaceae con 29. En conjunto, ellas comprenden $41 \%$ de la flora (cuadro 2). Leguminosae fue la familia más importante, tanto por el número de géneros como por el de especies. Sin embargo, a diferencia de lo encontrado en el área aledaña de Zimatán, la familia Asteraceae (Salas-Morales et al., 2003) no le sigue en importancia, ya que esta posición correspondió a Euphorbiaceae, mientras que Asteraceae obtuvo el tercer lugar. Esta secuencia es una caracterísitica compartida con otros sitios de la costa mexicana del Oceáno Pacífico (Lott, 1985; Trejo y Dirzo, 2002; Gordon et al., 2004).

Los géneros principales, por tener los mayores números de especies, fueron Ipomoea con 22 especies, Bursera (14), Senna (13), Croton (11), Cordia y Euphorbia (9 cada uno), y Randia y Solanum (8 cada uno). Hasta el momento se ha reconocido una especie nueva para la ciencia del género Bursera. En general, se aprecia un patrón de riqueza florística similar al reportado para el área de Zimatán (SalasMorales et al., 2003), con algunas excepciones como las familias Poaceae, Cyperaceae y Asteraceae.

Las especies presentes en el PNH que tienen algún sta tus de conservación según la NOM-ECOL-059 (SEMARNAT, 2002) son 14 y éstas se presentan en el cuadro 3. Cabe destacar que todas las especies de mangle se encuentran bajo protección especial y en el PNH tienen una distribución muy restringida, por lo que éstas deben ser un foco de atención prioritario. Bravaisia integerrima es otra especie de hábitat muy restringido, pero su área distribución en esta región no ha sido documentada dentro del $\mathrm{PNH}$, sino en la cuenca baja del río Cacaluta, por lo cual es muy importante que en un futuro cercano esta zona se integre al área natural protegida. Barkeria schoemakeri es una orquídea cuya población cuenta con unos cuantos individuos en una pequeña área y debiera ser otra de las especies prioritarias para su conservación dentro del PNH. Esta especie ocupa un área muy cercana a la carretera costera No. 200, por donde es muy fácil acceder y de donde actualmente se extrae arena del río Cacaluta y leña de la selva, lo cual pone en peligro a esta pequeña población de orquídeas.

Vegetación. Se reconocieron ocho comunidades vegetales en el PNH: (1) selva baja caducifolia, (2) selva baja caducifolia espinosa, (3) selva mediana subcaducifolia, (4) manglar, (5) sabana, (6) vegetación de dunas costeras, (7) bosque de galería y (8) vegetación secundaria. A continuación se ofrecen descripciones de estas comunidades.

Selva baja caducifolia. Es la vegetación dominante del PNH y en ella crecen casi $95 \%$ de las especies reportadas para toda el área de estudio. La altura del dosel oscila entre 6 y $8 \mathrm{~m}$, aunque algunas especies llegan a medir $16 \mathrm{~m}$. El estrato arbustivo alcanza hasta $5 \mathrm{~m}$ de altura; abundan las hierbas y los bejucos delgados. La familia dominante es Leguminosae, aunque la presencia de las especies de Bursera es bastante conspicua. En algunas cañadas húmedas este tipo de vegetación entra en contacto con la selva mediana subcaducifolia y en las márgenes de las corrientes de agua con el bosque de galería, llegando inclusive a mezclarse las especies de estos diferentes tipos de vegetación.

Debido a que se presenta en diferentes ambientes, esta vegetación es variable en su estructura y riqueza florística. Entre los árboles dominantes se encuentran Leucaena lan ceolata, Lonchocarpus lanceolatus, Lysiloma microphy llum, Piptadenia obliqua, Bursera excelsa, Jacaratia mexi cana, Cordia elaeagnoides y Crateva tapia, y acompañando a éstas se puede mencionar a Havardia campylacanthus, Piscidia carthagenensis, Senna atomaria, Bursera arbo - 
Cuadro 3. Especies listadas en la NOM-059- ECOL-2001 presentes en el Parque Nacional Huatulco, Oaxaca.

\begin{tabular}{lll}
\hline Familia & Nombre científico & Status \\
\hline Acanthaceae & Bravaisia integerrima & Amenazada \\
Bignoniaceae & Tabebuia chrysantha & Amenazada \\
Burseraceae & Bursera arborea & Amenazada \\
Cactaceae & Stenocereus chacalapensis & Protección especial, endémica \\
Combretaceae & Conocarpus erectus & Protección especial \\
Combretaceae & Laguncularia racemosa & Protección especial \\
Chrysobalanaceae & Licania arborea & Amenazada \\
Euphorbiaceae & Sapium macrocarpum & Amenazada \\
Fabaceae & Dalbergia congestiflora & Peligro de extinción \\
Fabaceae & Dalbergia granadillo & Peligro de extinción \\
Orchidaceae & Barkeria schoemakeri & Protección especial, endémica \\
Rhizophoraceae & Rhizophora mangle & Protección especial \\
Verbenaceae & Avicennia germinans & Protección especial \\
Zygophyllaceae & Guaiacum coulteri & Protección especial \\
\hline
\end{tabular}

rea, B. heteresthes y B. laurihuertae. Además, Dalbergia congestiflora y D. granadillo se encuentran solamente en este tipo de vegetación y ambas son muy escasas. Aunque Gordon et al. (2005) mencionan que Guaiacum coulteri ha sido extraída intensivamente de la región desde la década de los años 50 del siglo pasado, esta especie es medianamente abundante en el PNH.

En el estrato arbustivo se puede encontrar a Capparis verrucosa, Cnidoscolus multilobus, Croton niveus, Diospyros salicifolia, Euphorbia schlechtendalii, Hyperbaena mexicana, Malpighia ovata y Zapoteca formo $s a$; entre las hierbas y los bejucos se puede citar a Adenopodia oaxacana, Antigonon flavescens, Arrabidaea mollissima, Cardiospermum halicacabum, Ipomoea brac teata, Blechum pyramidatum, Chamaecrista rotundifolia, Holographis leticiana y Aristolochia nelsonii, entre muchas otras.

Selva baja caducifolia espinosa. Colindando con la vegetación de dunas costeras tierra adentro y en suelos arenosos, se encuentra la selva baja caducifolia espinosa. Los árboles que la componen tienen en promedio entre 4 y $5 \mathrm{~m}$ de altura, aunque algunos alcanzan hasta $6 \mathrm{~m}$, y son espinosos. Las especies más características de esta vegetación pertenecen a la familia Leguminosae, entre las que destacan Acacia cochliacantha y Prosopis juliflora, además de miembros de otras familias como Ziziphus amole, Cnidoscolus tubulosus, Jatropha sympetala y Bumelia celastrina. Acompañando a éstas se encuentran representantes de la familia Cactaceae: Opuntia tehuantepecana, Pereskiopsis kellermanii. Destacan también Cascabela ovata, Plumeria rubra y Randia laevigatoides. Cabe mencionar la abundancia de especies de la familia Burseraceae: Bursera cinerea, B. excelsa, B. instabilis y B. schlechtenda - lii, entre otras. Por otra parte, en el estrato arbustivo se encuentran Capparis indica, Pereskiopsis kellermanii, Melochia tomentosa, Diospyros aequoris, Pisonia aculea ta, Crossopetalum uragoga, Guettarda elliptica y Jacquinia donnell-smithii. Las hierbas y bejucos más conspicuos son Rivina humilis, Justicia caudata, Okenia hypo gaea, Boerhavia erecta, Cissus sicyoides, Antigonon fla vescens y Cardiospermum halicacabum. En la bahía de Cacaluta algunos árboles están cubiertos por la epífita Tillandsia maritima, que llega a alcanzar hasta $1.5 \mathrm{~m}$ de altura con todo y la inflorescencia.

Selva mediana subcaducifolia. Este tipo de vegetación se desarrolla básicamente en las pequeñas cañadas en las que la humedad relativa es mayor, tanto en los suelos como en el aire. Algunas cañadas mantienen una pequeña corriente de agua durante más tiempo que los arroyos, cuyo lecho se encuentra más expuesto a la intemperie. Algunas especies presentes en esta comunidad también se encuentran en el bosque de galería. Las especies dominantes alcanzan hasta $15 \mathrm{~m}$ de altura, como Homalium trichostemon y Lonchocarpus guatemalensis. Otras especies que las acompañan son Hippomane mancinella, Bravaisia integerrima y Andira inermis. A diferencia de la selva mediana subcaducifolia descrita por Salas-Morales et al. (2003) para la región de Zimatán, en el PNH Astronium graveolens, Inga vera e I. paterno, todas ellas especies propias de climas más húmedos o de las márgenes de corrientes permanentes, no fueron halladas.

Manglar. Esta comunidad que puede reconocerse claramente por las especies que la componen, se encuentra asociada de manera compleja a las dunas costeras, a la selva baja caducifolia espinosa y a la selva baja caducifolia en las 
desembocaduras de ríos y en las pequeñas depresiones que se forman a la orilla del mar. Las especies más conspicuas son Conocarpus erectus, Avicennia germinans y Rhizophora mangle; sin embargo, hay otras especies que le dan una fisonomía particular a esta vegetación. Entre los árboles se puede mencionar a Recchia mexicana, Caesalpinia velutina, Jatropha mexicana, Achatocarpus gracilis y Diospyros aequoris. También se observan elementos arbustivos como Crossopetalum uragoga, Casearia corymbosa, Chiococca alba, así como hierbas y bejucos, sobre todo en ambientes ubicados más tierra adentro: Combretum fruticosum, Ipomoea microsepala, Jouvea pilosa, Trixis silvatica y Tournefortia densiflora.

Sabana. Esta comunidad se distribuye en las cimas de pequeños lomeríos situados en el norte del PNH. Fisonómicamente se trata de una vegetación abierta con escasos árboles y hierbas, a diferencia de las que se encuentran en Nizanda, donde dominan hierbas amacolladas de la familia Poaceae (Pérez-García et al., 2001; López-Olmedo et al., 2006, 2007). Esta vegetación es similar en su fisonomía a las comunidades reportadas para la zona de Zimatán (Salas-Morales et al., 2003); sin embargo, es notoria la ausencia de Pinus maximinoi, Psidium guajava e Hymenaea courbaril, lo cual sin duda se debe a que este tipo de vegetación se encuentra a mayores altitudes en la zona de Zimatán. La sabana está dominada por plantas herbáceas, mientras que los árboles y arbustos que allí crecen forman conjuntos compactos a manera de islas en medio de las hierbas. Entre los árboles se distinguen claramente Curatella americana, Coccoloba liebmannii y Byrsonima crassifolia, y asociados a éstos se pueden mencionar a Schoepfia schreberi, Diospyros aequoris, Acacia collinsii y Bursera fagaroides. Los arbustos más abundantes son Krameria cuspidata, $K$. revoluta, Croton suberosus e Indigofera platycarpa. Entre las hierbas observamos a Mitracarpus rhadinophyllus, Chamaecrista hispidula y Aniseia martinicensis.
Vegetación de dunas costeras. Se localiza en suelos evidentemente arenosos y se pueden distinguir básicamente dos cinturones angostos que se encuentran en una franja a la orilla del mar. El primero, más cercano a la línea de costa, está compuesto principalmente por Ipomoea pes-caprae y manchones amacollados de Jouvea pilosa. El segundo cinturón está compuesto por hierbas anuales y algunos arbustos pequeños, entre los que destacan Crossopetalum urago ga, Salpianthus arenarius, Chiococca sp., Guettarda ellip tica, Tournefortia volubilis y T. densiflora. Junto con estas especies se pueden encontrar algunos individuos achaparrados de especies arbóreas, como Gliricidia sepium, Vitex mollis, Bursera excelsa, Prosopis juliflora, que dan un aspecto peculiar a esta vegetación.

Bosque de galería. Este tipo de vegetación es difícil de caracterizar debido a su heterogeneidad. Sin embargo, es fácil distinguirlo por mantenerse siempre verde, ya que se ubica en franjas delgadas a lado de los ríos y por su altura, pues allí los árboles alcanzan hasta $12 \mathrm{~m}$. Las especies más conspicuas son Astianthus viminalis, Licania arborea, Sapium macrocarpum, Homalium trichostemon y Vitex mollis. En algunas desembocaduras de estas corrientes de agua las especies de esta comunidad se mezclan con las especies de manglar.

Vegetación secundaria. Sin duda estas comunidades son inducidas por la actividad agrícola y ganadera en el PNH y sus alrededores, así como por el uso que históricamente hubo en el área. La vegetación secundaria se ha derivado básicamente de la selva baja caducifolia y de la selva mediana subcaducifolia, las cuales se encontraban en lomeríos suaves o en planicies, donde los suelos son un poco más profundos y poseen mayor humedad. Aunque la presión sobre la vegetación ha disminuido, todavía existen comunidades humanas dentro de los límites del PNH que llevan a cabo actividades agrícolas y ganaderas, como en la cuenca baja del río Cacaluta. Las principales especies que

Cuadro 4. Comparación de la riqueza florística del Parque Nacional Huatulco y otras regiones estudiadas.

\begin{tabular}{|c|c|c|c|c|c|}
\hline Región y referencia & Superficie $\left(\mathrm{km}^{2}\right)$ & No. familias & No. géneros & No. especies & No. registros \\
\hline $\begin{array}{l}\text { Depresión Central, Chiapas } \\
\text { (Reyes-García y Sousa, 1997) }\end{array}$ & 9000 & 103 & 489 & 998 & 1261 \\
\hline $\begin{array}{l}\text { Distrito de Tehuantepec, Oaxaca } \\
\text { (Torres-Colín et al., 1997) }\end{array}$ & 6600 & 154 & 776 & 1720 & 8500 \\
\hline $\begin{array}{l}\text { Costa Grande, Guerrero } \\
\text { (Peralta-Gómez et al., 2000) }\end{array}$ & 2500 & 135 & 527 & 1047 & 3020 \\
\hline $\begin{array}{l}\text { Región de Zimatán, Oaxaca } \\
\text { (Salas-Morales et al., 2003) }\end{array}$ & 713 & 144 & 668 & 1384 & 8063 \\
\hline $\begin{array}{l}\text { Parque Nacional Huatulco, Oaxaca } \\
\text { (Este trabajo) }\end{array}$ & 63 & 91 & 391 & 736 & 3312 \\
\hline
\end{tabular}


se observan en las comunidades secundarias son Guazuma ulmifolia, Mimosa acantholoba, M. antioquensis, Cnidoscolus multilobus, Senna uniflora y Mentzelia aspe $r a$, entre otras. En el área que ocupa la laguna El Zanate, en la cuenca baja del río Cacaluta, cuando se seca o no se llena durante la época de lluvias, crece un estrato de hierbas como Heliotropium curassavicum y Aniseia martinicensis, y otro arbustivo con especies como Mimosa pigra y Salpianthus purpurascens, que desaparecen una vez que la laguna se llena de agua.

\section{Discusión}

El trabajo de recolección de plantas realizado en el PNH ha permitido mostrar una relevante riqueza florística en comparación con otras regiones estudiadas, a pesar de contar con un territorio pequeño (cuadro 4). Probablemente la causa de esto sea la heterogeneidad de hábitats y asociaciones vegetales encontrados en un estre cho intervalo altitudinal.

De acuerdo con el estudio de Castillo-Campos et al. (1997), la riqueza florística de su área de estudio, que incluye al PNH, consistía en 78 familias, 272 géneros y 413 especies. Gracias a una mayor intensidad de colecta y a las determinaciones taxonómicas, en el presente trabajo se reportan 91 familias, 391 géneros y 736 especies para una superficie de casi la décima parte del área cubierta $(51,510$ ha) por el estudio mencionado. Esto significa que se ha incrementado el conocimiento florístico del PNH en $17 \%$ para el nivel de familia, en $44 \%$ para el de género y en $78 \%$ para el de especie, Además, es esperable que esto números aumentarán una vez que se determine todo el material aún no identificado. Estos resultados revelan la importancia de los inventarios florísticos intensivos realizados en áreas pequeñas.

Cabe mencionar la riqueza de especies de Bursera encontradas en el PNH: 13 previamente conocidas más una especie nueva, cifras que llaman la atención por su semejanza con las 13 especies reportadas por Salas-Morales et al. (2003) para un área 11 veces más grande que el PNH. Por otra parte, de la familia más abundante (Leguminosae), en el PNH se encuentran más de la mitad de las especies reportadas para la región de Zimatán. La intensidad de colecta también permite afirmar con cierta confianza que muy probablemente la región constituye el límite noreste de la distribución geográfica de la especie Holographis leti ciana (Acanthaceae).

En la selva baja caducifolia y en el PNH en general se puede encontrar a Achatocarpus oaxacana (endémica de las costas de Oaxaca y sureste de Honduras) y a Jatropha sympetala, Caesalpinia hughesii, Lonchocarpus emargina tus, Randia cinerea, Recchia mexicana, Sapranthus viola ceus, todas ellas endémicas de los bosques secos de la costa mesoamericana del Pacífico (Lott y Atkinson, 2002, 2006;
Gordon et al., 2004). Por su parte, Bucida macrostachya, Croton axillaris, Gyrocarpus mocinnoi, Havardia campy lacanthus, Heliocarpus occidentalis, Hyptis tomentosa, Lagrezia monosperma, Lonchocarpus constrictus, Mimosa robusta y Torrubia macrocarpa son de distribución mesoamericana (Gordon et al., 2003). Esta información sugiere que el PNH puede funcionar como un reservorio de material genético de éstas y otras especies, en caso de que otros sitios de la región sufrieran afectaciones de la cubierta vegetal a gran escala. La selva baja caducifolia del PNH alberga especies que no se encuentran en otro tipo de vegetación, además de especies que por su escasez se pueden clasificar como raras.

La presencia de algunas especies como Hura polyandra y Lonchocarpus guatemalensis en altitudes por debajo de 200 m s.n.m. permite inferir un posible gradiente de humedad que va de este a oeste en la costa este de Oaxaca, tal como fue sugerido por Salas-Morales (2002), ya que estas especies en el área vecina de Zimatán sólo se desarrollan en áreas de mayor altitud.

El PNH alberga a 14 especies enlistadas en la NOM059-ECOL-2001 (SEMARNAT, 2002), lo cual resalta aún más la importancia del área. Al respecto, cabe mencionar que Gordon et al. (2005) propusieron que la especie Guaiacum coulteri fuera colocada en la categoría 'Amenazada' con base en los criterios A2c y d de la IUCN, debido a su historia de explotación y al deterioro de su hábitat. Sin embargo, en el PNH la especie es medianamente abundante y en el área de Zimatán se pueden encontrar individuos de hasta $20 \mathrm{~m}$ de altura.

La zona donde se encuentra el PNH es reconocida como centro de distribución de endemismos de los bosques tropicales secos (Lott y Atkinson, 2006). Además, la región Costa de Oaxaca ha sido destacada como un área de importancia para la conservación biológica (Gordon et al., 2004; Cué-Bär et al., 2006). Estudios recientes a partir de modelos coremáticos ponen en evidencia la importancia estratégica de la microcuenca del río Cacaluta para la conservación de la riqueza natural del Parque (Gómez-Rojo et al., 2006).

\section{Agradecimientos}

Expresamos un profundo agradecimiento al personal del Herbario Nacional (MEXU), en especial a Mario Sousa y Gerardo Salazar, por el apoyo proporcionado al trabajo florístico realizado por SERBO en el estado de Oaxaca. Agradecemos la valiosa ayuda de los especialistas de las diferentes familias incluidas en el listado y a otros botánicos: S. Acosta (Acanthaceae), C. Anderson (Malpighiaceae), W. Anderson (Malpighiaceae), G. Andrade (Leguminosae), S. Arias (Cactaceae), F. Barrie (Myrtaceae), A. Borhidi (Rubiaceae), A. Bornstein (Piperaceae), J. Calónico (Sapindaceae), E. Carranza 
(Convolvulaceae), J. Castrejón (Cucurbitaceae), F. Chiang (varias familias), R. Cruz (Leguminosae), T. Daniel (Acanthaceae), A. Delgado (Leguminosae), N. Diego (Cyperaceae), A. Espejo (Commelinaceae y Bromeliaceae) G. Flores (Leguminosae), J.C. Flores (Moraceae), P. Fryxell (Malvaceae), M.R. García (Lamiaceae), C. Granados (Bromeliaceae), R. Grether (Leguminosae), M.A. Guadarrama (Commelinaceae), H. Hernández (Leguminosae), G. Ibarra (Moraceae), J. Jiménez (Euphorbiaceae), V. Juárez (Asclepiadaceae), G. Juárez (varias familias), E. León (Malpighiaceae), E. Lira (Boraginaceae), A.R. López-Fe rra ri (Commelinaceae y Bromeliaceae), E. Lott (varias familias), L. Lozada (Asclepiadaceae), M. Martínez (Euphorbiaceae), E. Martínez (Bignoniaceae y otras), J. Mickel (Pteridophyta), M. Mora (varias familias), G. Nesom (Asteraceae), G. Pilz (Nyctaginaceae), A. Ponce (Malvaceae), F. Ramos (Asteraceae y otras), C. Ramos (Rutaceae y otras), J.R Reeder y C.G. Reeder (Poaceae), L. Rico (Leguminosae), A. Rodríguez (Solanaceae), J. Rzedowski (Burseraceae), G. Salazar (Orchidaceae), G. Schatz (Annonaceae), M. Sousa (Leguminosae), V. Steinmann (Euphorbiaceae y Urticaceae), S. Sumaya (Amaranthaceae), C. Taylor (Rubiaceae), O. Téllez (Dioscoreaceae), L. Torres (Leguminosae), B.L. Turner (Asteraceae), K. Velasco (varias familias) y J.L. Villaseñor (Asteraceae). Un especial agradecimiento a Tom Wendt (herbario TEX), por las determinaciones de diversas familias y su ayuda para distribuir material en Estados Unidos. Emily Lott, apasionada de la botánica mexicana, colaboró con el trabajo de herbario y nos ha abierto el canal de comunicación con otros botánicos. A Rodrigo Duno y Ramón Cuevas agradecemos su tiempo y sus atinadas observaciones a una versión previa del manuscrito. A. Sánchez, G. Juárez, K. Velasco, E. Vásquez, A. Ruiz, M. Hernández, M. Mendoza, F. Maldonado y B. López colaboraron en el trabajo de campo. Este proyecto recibió apoyo económico de la Comisión Nacional para el Conocimiento y Uso de la Biodiversidad (FB1021/BK0004/04) y de la Comisión Nacional de Áreas Naturales Protegidas a través de la Dirección del Parque Nacional Huatulco y del Fondo Oaxaqueño para la Conservación (FOCN-04'2006).

\section{Literatura citada}

Arriaga L., Espinoza J.M., Aguilar C., Martínez E., Gómez L. y Loa E. (Coords.) 2000. Regiones Terrestres Prioritarias de México. Comisión Nacional para el Conocimiento y Uso de la Biodiversidad, México, D.F.

Castillo-Campos, G., Moreno-Casasola P., Medina M.E. y Zamora P. 1997. Flora y vegetación de las bahías de Huatulco, Oaxaca, México. Ciencia y Mar 1: 3-44.

CETENAL-UNAM [Comisión de Estudios del Territorio Nacional-Universidad Nacional Autónoma de México]. 1970. Carta de Climas San Pedro Pochutla 14P-(II) Oaxaca 14Q-VII.
México, D.F.

CONANP [Comisión Nacional de Áreas Naturales Protegidas]. 2003. Programa de Manejo del Parque Nacional Huatulco. Dirección General de Manejo para la Conservación, CONANP. México, D.F.

Cué-Bär E.M., Villaseñor J.L., Morrone J.J. e Ibarra-Manríquez G. 2006. Identifying priority areas for conservation in Mexican tropical deciduous forest on tree species. Interciencia 31:712719.

Cronquist A. 1981. An Integrated System of Classification of Flowering Plants. Columbia University Press, Nueva York.

Escalona I. 1996. Caracterización de los humedales costeros de Santa María Huatulco, Oaxaca. Informe del Proyecto Conservación y Desarrollo de la Costa de Oaxaca, Comisión Oaxaqueña de Defensa Ecológica-Fondo Mundial para la Naturaleza, Oaxaca.

Ferrusquía-Villafranca I. 1998. Geología de México: una sinopsis. En: Ramamoorthy T.P., Bye R., Lot A. y Fa J. (Comps.) Diversidad Biológica de México: Orígenes y Distribución, pp. 3-108, Universidad Autónoma de México, México, D.F.

García E. 2004. Modificaciones al sistema de clasificación climá tica de Köppen. $5^{\text {a }}$ ed., Universidad Nacional Autónoma de México. Mexico, D.F.

García G., Salas S.H., Schibli L., Aguilar R., Acosta S. y Salazar A. 1992. Análisis de la vegetación y uso actual del suelo en el estado de Oaxaca, Fase I (Costa y Sierra Sur). Informa Técnico presentado a WWF. SERBO, A.C. Oaxaca. [Inédito]

García-Mendoza A. 2004. Integración del conocimiento florístico del estado. En: García-Mendoza A.J., Ordóñez M.J. y BrionesSalas M. (Eds.) Biodive rsidad de Oaxaca, pp. 305-325, Universidad Nacional Autónoma de México - Fondo Oaxaqueño para la conservación de la Naturaleza-WWF, México, D.F.

García-Mendoza, A. y Torres-Colín R. 1999. Estado actual del conocimiento sobre la flora de Oaxaca. En: Vásquez-Dávila M.A. Ed. Sociedad y Naturaleza en Oaxaca 3: Vegetación y Flora, pp. 50-86, Instituto Tecnológico Agropecuario de Oaxaca, Oaxaca.

Gómez-Rojo V.R., Domínguez-Licona J.M. y GonzálezHernández. T.D. 2006. Análisis territorial de la micro-cuenca y bahía del río Cacaluta, Santa María Huatulco, Oaxaca. Investigaciones Geográficas, UNAM. 60:22-45.

González, M.A. y Miranda M.E. 1994. Estimación preliminar de la biodiversidad del municipio de Santa María Huatulco, Oaxaca. Grupo Autónomo para la Investigación Ambiental (GAIA. A.C.), Oaxaca, México. [Documento inédito].

Gordon, J.E., Barrance A.J. y Schreckenberg K. 2003. Are rare species useful species? Obstacles to the conservation of tree diversity in the dry forest zone agro-ecosystems of Mesoamerica. Global Ecology and Biogeography 12:13-19.

Gordon J.E., Hawthorne W.D., Reyes-García A., Sandoval G. y Barrance A.J. 2004. Assessing landscapes: a case study of tree and shrub diversity in the seasonally dry tropical forests of Oaxaca, Mexico and southern Honduras. Biological Conservation 117:429-442.

Gordon J.E., González M.A., Hernández J.V., Lavariega R.O. y Reyes-García A. 2005. Guaiacum coulteri: an over-logged dry forest tree of Oaxaca, Mexico. Oryx 39: 82-85.

INEGI [Instituto Nacional de Estadística, Geografía e Informática]. 1980. Carta de climas Villahermosa. Escala 1:1, 
000,00. México, D.F.

INEGI. 1985. Carta de Aguas Subterráneas y Aguas Superficiales D14-3 Puerto Escondido. Escala 1:250, 000. México, D.F.

INEGI. 1988a. Carta Geológica D14-3 Puerto Escondido. Escala 1:250, 000. México, D.F.

INEGI. 1988b. Carta Topográfica D14-3 Puerto Escondido. Escala 1:250, 000. México, D.F.

INEGI. 1989. Carta Edafológica D14-3 Puerto Escondido. Escala 1:250, 000. México, D.F.

López-Olmedo L.I., Meave J.A. y Pérez-García E.A. 2007. Floristic and structural contrasts between natural savannas and anthropogenic pastures in a tropical dry landscape. The Rangeland Journal 29:181-190.

López-Olmedo, L.I., Pérez-García E.A. y Meave J.A. 2006. Estructura y composición florística de las sabanas de la región de Nizanda, Istmo de Tehuantepec (Oaxaca), México. Acta Botanica Mexicana 77:41-67.

Lorence D.H. y García-Mendoza A. 1989. Oaxaca, México. En: Campbell D.G. y Hammond H.D. Eds. Floristic Inventory of Tropical Countries: the Status of Plant Systematic, Collections, and Vegetation, plus Recommendations for the Future, pp. 253269, New York Botanical Garden. Nueva York.

Lott E.J. 1985. Listados Florísticos de México III. La Estación de Biología Chamela, Jalisco. Instituto de Biología, Universidad Nacional Autónoma de México, México, D.F.

Lott E.J. y Atkinson T.H.. 2002. Biodiversidad y fitogeografía de Chamela-Cuixmala, Jalisco. En: Noguera F.A., Vega-Rivera J.H., García-Aldrete A.N. y Quesada-Avendaño M. Eds. Historia Natural de Chamela, pp. 83-97, Universidad Nacional Autónoma de México, México, D.F.

Lott E.J. y Atkinson T.H. 2006. Mexican and Central American seasonally dry tropical forests: Chamela-Cuixmala, Jalisco, as a focal point. En: Pennington R.T., Lewis G.P. y Ratter J.A. Eds. Neotropical Savannas and Seasonally Dry Forests: Plant Diversity, Biogeography, and Conservation, pp. 315-342, CRC, Taylor and Francis, Boca Raton, Florida.

Mickel J.T. y Beitel J.M. 1988. Pteridophyte flora of Oaxaca, Mexico. Memoirs of the New York Botanical Garden 46:1-568.

Miranda F. y Hernández-X. E. 1963. Los tipos de vegetación de México y su clasificación. Boletín de la Sociedad Botánica de México 28:29-179.

Morales H. 1998. Análisis del paisaje en el corredor turístico de Bahías de Huatulco. Tesis de Licenciatura en Geografía. Facultad de Filosofía y Letras. Universidad Nacional Autónoma de México, México, D.F., 145 pp.
Peralta-Gómez S., Diego N. y Gual-Díaz M.. 2000. Listados flo rísticos de México XIX. La Costa Grande de Guerrero. Instituto de Biología, Universidad Nacional Autónoma de México, México, D.F.

Pérez-García E.A., Meave J. y Gallardo C. 2001. Vegetación y flora de la región de Nizanda, Istmo de Tehuantepec, Oaxaca, México. Acta Botanica Mexicana 56:19-88.

Reyes-García A. y Sousa M. 1997. Listados florísticos de México XVII. Depresión Central de Chiapas. La Selva Baja Caducifolia. Instituto de Biología, Universidad Nacional Autónoma de México. México, D.F.

Rzedowski J. 1991. Diversidad y orígenes de la flora fanerogámica de México. Acta Botanica Mexicana 14:3-21.

Salas-Morales S.H. 2002. Relación entre la heterogeneidad ambiental y la variabilidad estructural de las selvas tropicales secas de la costa de Oaxaca, México. Tesis de Maestría, Facultad de Ciencias, Universidad Nacional Autónoma de México, México, D.F., 101 pp.

Salas-Morales S.H., Saynes-Vásquez A. y Schibli L.. 2003. Flora de la costa de Oaxaca: lista florística de la región de Zimatán. Boletín de la Sociedad Botánica de México 72:21-58.

SEMARNAT [Secretaría del Medio Ambiente y Recursos Naturales]. 2002. Norma Oficial Mexicana NOM-059-ECOL2001, Protección ambiental-Especies nativas de México de flora y fauna silvestres-Categorías de riesgo y especificaciones para su inclusión, exclusión o cambio-Lista de especies en riesgo. Diario Oficial de la Federación. 2a Sección, 6 de marzo de 2002.

Toledo V.M. 1988. La diversidad biológica de México. Ciencia y Desarrollo 14:17-30.

Torres-Colín R., Torres-Colín L., Dávila-Aranda P. y Villaseñor J.L.. 1997. Listados florísticos de México XVI. Flora del Distrito de Tehuantepec, Oaxaca. Instituto de Biología, Universidad Nacional Autónoma de México, México, D.F.

Torres-Colín R. 2004. Tipos de vegetación. En: García-Mendoza A.J., Ordóñez M.J. y Briones-Salas M. Eds. Biodiversidad de Oaxaca, pp. 105-117, Universidad Nacional Autónoma de México-Fondo Oaxaqueño para la Conservación de la Naturaleza-World Wildlife Fund, México, D.F.

Trejo I. 1998. Distribución y diversidad de selvas bajas de México: relaciones con el clima y el suelo. Tesis de Doctorado. Universidad Nacional Autónoma de México, Mexico, D.F.

Trejo I. y Dirzo R. 2002. Floristic diversity of Mexican seasonally dry tropical forests. Biodiversity and Conservation 11:20632084.

Recibido: 8 de agosto de 2007

Aceptado: 7 de noviembre de 2007 
Apéndice 1. Lista de plantas vasculares de la Costa de Oaxaca (2): Parque Nacional Huatulco. Se presenta la siguiente información para cada especie incluida en la lista florística: nombre científico, forma de crecimiento, mes o meses de floración o fructificación, tipo de vegetación en el que se recolectó, colector y número de colecta. A continuación se presentan los nombres completos y las abreviaturas utilizadas para los tipos de vegetación y para los colectores: tipos de vegetación; selva baja caducifolia SBC; selva baja caducifolia espinosa SBCE; selva mediana subcaducifolia SMS; bosque de galeria Ga; manglar Ma; sabana Sa; vegetación de dunas costeras VD; pastizal Pz; el número 2 después del tipo de vegetación indica vegetación secundaria. Colectores: Alejandrino de Ávila Hernández AÁH; Arturo Nava Zafra ANZ; Arturo Sánchez Martínez ASM; Gonzálo Juárez García GJ; Kenia Velasco Gutiérrez KV; Silvia H. Salas Morales SS; Alfredo Saynes Vásquez SAY; Misael Elorsa Castillo ME. Las especies marcadas con $\neq$ son introducidas o cultivadas.

\section{PTERIDOPHYTA}

\section{ADIANTACEAE}

Adiantum amplum C.Presl. Hierba 1 m. Fértil. Ene. SBC, oriIla de arroyo. KV 691.

\section{POLYPODIACEAE}

Cheilanthes lozanoi (Maxon) R.M.Tryon et A.F.Tryon. Hierba 25-30 cm. Soros cafés. Ago. SBC. ASM 906; GJ 2644.

\section{SCHIZAEACEAE}

Lygodium venustum Sw. Hierba escandente o trepadora. Soros. Sept.-feb. SBC, SMS, Ga. ANZ 939; SAY 4199, 5186; KV 668; ASM 605.

\section{SELAGINELLACEAE}

Selaginella sertata Spring. Hierba rupícola. Estéril. Oct. Orilla de río. SAY 5189.

\section{MAGNOLIOPHYTA MAGNOLIOPSIDA}

\section{ACANTHACEAE}

Aphelandra scabra (Vahl) Sm. Arbusto 1-3 m. Fl roja. Nov.mar. SBC. ME 6443; GJ 2204; ANZ, 726; SS 5483, 5538.

Blechum pyramidatum (Lam.) Urb. Hierba 10-80 cm. Fl blanca a lila. Oct.-feb. SBC. ANZ 782, 901; SS 5497; ASM 1258, 1383.

Bravaisia integerrima (Spreng.) Standl. Árbol 3-12 m. Fl blanca con centro morado. Feb.-abril. SMS. ME 6478; ASM 1297,1408,1440; SAY 4372.

Carlowrightia arizonica A.Gray. Hierba 50 cm-1 m. Fl blanca. Feb. SBC. ANZ 829, 969.

Chileranthemum pyramidatum (Lindau) T.F.Daniel. Hierba 50 cm-1 m. Fl morada. Sept. SBC. ASM 1070,1073.

Elytraria imbricata (Vahl) Pers. Hierba 10-40 cm. Fl blanca. Sept.-ene. SBC ANZ 1219, 1246b, 443; SS 5492; ASM 203.

Henrya insularis Nees. Hierba 20-70 cm. Fl blanca. Ene.-jun. SBC. ASM 1217, 1391, 629; SAY 5451, 5504.

Holographis leticiana T.F.Daniel. Hierba $1 \mathrm{~m}$. FI roja. Dic. SBC. ANZ 750.

Justicia candicans (Nees) L.D.Benson. Arbusto 30 cm-2.5 m.
FI roja. Ago.-dic. SBC. ANZ 777; SS 5482, 5535; ASM 1052, 136.

Justicia carthaginensis Jacq. Hierba 40-70 cm. Fl lila a morada. Oct.-nov. SBC. ASM 397, 430; SAY 5013, 5029, 5234.

Justicia caudata A.Gray. Arbusto $30 \mathrm{~cm}-2 \mathrm{~m}$. Fl rosa, lila o morada. Sept.-dic. SBC, SBC2, SBCE. ME 7280, 7353; ANZ $1155,1193,481$.

Justicia ramosa (Oerst.) V.A.W.Graham. Hierba 30-60 cm. FI lila, rosa o guinda. Ene.-may. SBC. ASM 563, 635, 826; SAY 5426, 5431.

Justicia sp. Hierba 30 cm. FI morada. Sept. SBC. SAY 4251.

Ruellia foetida Willd. Arbusto $30 \mathrm{~cm}-1.5 \mathrm{~m}$. Fl blanca. Nov.mar. SBC. GJ 2045, ANZ 1295, SS 5532; ASM 1221, 1257.

Ruellia galeottii Leonard. Hierba $25 \mathrm{~cm}$. FI lila. Feb. SBC. ASM 1259.

Ruellia inundata Kunth. Hierba $30 \mathrm{~cm}-1 \mathrm{~m}$. Fl lila o morada. Nov.feb. SBC. GJ 1955; ANZ 1296, 728, 831a; SS 5468.

Tetramerium nervosum Nees. Hierba 20-60 cm. FI blanca. Oct.-jun. SBC. GJ 2193, 2203; ANZ 486, 754; ASM 361.

ACHATOCARPACEAE

Achatocarpus gracilis H.Walter. Árbol o arbusto 3-5 m. FI blanca. Dic.-ene. SBC, VD. ANZ 801; SS 5608; ASM 1412, 641,679 .

Achatocarpus nigricans Triana. Árbol $6 \mathrm{~m}$. Fr verde. May. SBC2. ASM 808.

Achatocarpus oaxacanus Standl. Árbol o arbusto 4-6 m. Fr verde. Ene. SBC. ASM 417, 701; KV 673.

\section{AMARANTHACEAE}

Alternanthera flava (L.) Mears. Arbusto 1.5-2.5 m. Fl blanca. Nov.-dic. SBC. ANZ 1312; SS 5534; ASM 479.

Alternanthera pycnantha (Benth.) Standl. Hierba sufrutescente 50-80 cm. Fl blanca. Sept. SBC. ANZ 1194; ASM 648; SAY 5235 .

Amaranthus spinosus L. Hierba $20 \mathrm{~cm}-1.5 \mathrm{~m}$. Fl blanca. Abr.oct. SBC, SMS. AÁH 78; ASM 1114, 1432; SAY 4198bis, 5750. 
Amaranthus sp. Hierba $80 \mathrm{~cm}-1$ m. Fl verde. Ene. SBC. ANZ 792; KV 666.

Chamissoa acuminata Mart. var. no determinada. Hierba 40 cm. Fr café. Feb. SBC. ASM 1253.

Chamissoa acuminata Mart. var. swansonii Sohmer. Arbusto 80 cm-6 m. Fl blanca. Sept.-nov. SBC. ANZ 451, 581; ASM $78,343,429$.

Chamissoa altissima (Jacq.) Kunth. Bejuco. Fr verde. Abr. SBC. ASM 1439, 1446.

Gomphrena nitida Rothr. Hierba $20-30 \mathrm{~cm}$. Fl blanca o amarilla. Sept.-ene. SBC. ANZ 1199; ASM 579; SAY 4321.

Gomphrena sp. Hierba $20 \mathrm{~cm}$. Fl blanca con rojo y amarillo. Oct. SBC. ASM 18.

Iresine calea (Ibáñez) Standl. Hierba 50 cm-2 m. Fl blanca. Ene.-jun. SBC. ANZ 944; ASM 664; SAY 5572; KV 669.

Iresine interrupta Benth. Hierba o arbusto decumbente 80 cm. Fl blanca o amarilla. Feb. SBC. ASM 1227; SAY 5320, 5457.

Iresine nigra Uline et W.L.Bray. Arbusto 3 m. Fl blanca. Oct. SBC. SAY 5230.

Lagrezia monosperma (Rose) Standl. Arbusto 1-3 m. Fl blanca o verde. Oct.-ene. SBC. GJ 1750, 1850; ANZ 786; ASM $143,318$.

\section{ANACARDIACEAE}

Comocladia engleriana Loes. Árbol o arbusto 3-6 m. Fl roja o guinda. Oct.-feb. SBC, Ga. GJ 1893; ASM 560, 618; SAY 5214, 5351.

Spondias purpurea L. Árbol 3-10 m. Fl roja. Ene.-may. SBC, Ga, SMS. GJ 1816, 2046, 2175; ANZ 907, 979.

\section{ANNONACEAE}

Annona reticulata L. Árbol $12 \mathrm{~m}$. Fl amarillo con rojo. Abril. SBC. ANZ 998.

Annona squamosa L. Árbol o arbusto 2.5-7 m. Ago.-oct. SBC. G) 1738, 1855, 1882; ANZ 1056, 1118.

Sapranthus violaceus (Dunal) Saff. Árbol 15 m. Fl morada. Feb. SBC. ASM 1240; ANZ 1510; SS 5640; SAY 4416.

\section{APOCYNACEAE}

Cascabela ovata (Cav.) Lippold. Árbol o arbusto 2-10 m. FI amarilla. Jul.-oct. SBC, SBCE, SMS. AÁH. 66; GJ 1762; ANZ $1101,489$.

Cascabela thevetioides (Kunth) Lippold. Árbol $4 \mathrm{~m}$. Fl amariIla. Jun. SBC. ASM 1618.

Forsteronia spicata (Jacq.) G.Mey. Arbusto escandente o beju- co. FI amarilla. Jun. SBC. ANZ 1526; ASM 1610.

Haplophyton cimicidum A.DC. Arbusto $30 \mathrm{~cm}-1.5 \mathrm{~m}$. FI amarilla. May.-nov. SBC. ME 6974; ANZ 1092; SS 5892; ASM 1600, 1632.

Mandevilla subsagittata (Ruiz et Pav.) Woodson. Bejuco. FI amarilla. Jul. SBC. ASM 1817; SAY 5639.

Plumeria rubra L. Árbol 2-8 m. Fl blanca. May.-oct. SBC, SBCE. ANZ 1030, 1035, 740; SS 5895; ASM 1015.

Rauvolfia tetraphylla L. Árbol o arbusto $30 \mathrm{~cm}-3 \mathrm{~m}$. Fl blanca. May.-oct. SBC, SBCE, SMS. AÁH 84; GJ 2177, 2363; ANZ 1125, 1500.

Stemmadenia mollis Benth. Arbusto $2 \mathrm{~m}$. Fl amarilla. Abr. SBC. ASM 1360.

Stemmadenia obovata K.Schum. Árbol o arbusto 2-9 m. FI amarilla. Abr.-oct. SBC, Ga. GJ 1757, 1821, 2186; ANZ 1012, 1053.

Tabernaemontana amygdalifolia Jacq. Árbol o arbusto 2-3 m. FI blanca. Mar.-jul. SBC, SBCE. SS 5609, 5633, 5930; ASM 1613; SAY 5466.

Thevetia ahouai (L.) A.DC. Árbol 4 m. Fr. verde. Oct. SBC. ASM 103.

Urechites andrieuxii Müll.Arg. Bejuco. Fr verde. Dic. SBC. SS 5489.

\section{ARISTOLOCHIACEAE}

Aristolochia anguicida Jacq. Bejuco. Fr seco. Mar. SBC. GJ 2026.

Aristolochia nelsonii Eastw. Hierba rastrera. Fl guinda con blanco. Jul.-sept. SBC. ME 6982; ANZ 1126; ASM 972; SAY 4259.

ASCLEPIADACEAE

Dictyanthus sp. Bejuco. Fr verde. Abr. SBC. SAY 5395

Funastrum clausum (Jacq.) Schltr. Bejuco. Fl blanca. Oct.-dic. SBC. ANZ 733; ASM 259; SAY 5167.

Macroscepis diademata (Ker Gawl.) W.D.Stevens. Bejuco. FI verde-amarillenta a café. Nov.-ene. SBC. ANZ 553; KV 706.

Marsdenia callosa Juárez-Jaimes et W.D.Stevens. Bejuco. FI blanca. Ago.-oct. SBC. AÁH 56; ASM 153, 16.

Marsdenia coulteri HemsI. Bejuco. Fr verde. Oct. SBC. ASM 297.

Marsdenia lanata (Paul G.Wilson) W.D.Stevens. Bejuco. FI amarilla. Jul. SBC. ANZ 1309; SAY 5645.

Marsdenia trivirgulata Bartlett. Bejuco. Fl blanca. Ago. SBC. ANZ 1146 
Prosthecidiscus guatemalensis Donn.Sm. Bejuco. Fr verde. Ene. SBC. ASM 646.

\section{ASTERACEAE}

Adenophyllum aurantium (L.) Strother. Hierba $40 \mathrm{~cm}-1 \mathrm{~m}$. FI anaranjada. Ene. SBC. ANZ 835; ASM 582, 594; KV 702.

Ageratina crassiramea (B.L.Rob.) R.M.King et H.Rob. Arbusto 1.5. Fl café-amarilla. Mar. SBC. GJ 2044.

Baltimora geminata (Brandgee) Stuessy. Hierba $70 \mathrm{~cm}$. FI amarilla. Nov. SBC. ASM 366.

Bidens riparia Kunth. Hierba $80 \mathrm{~cm}$. Fr verde amarillento. Oct. SBC. ASM 213.

Blumea viscosa (Mill.) D'Arcy. Hierba $40 \mathrm{~cm}$. Fr café. Abr. SBC. ASM 1425.

Brickellia diffusa (Vahl) A.Gray. Hierba $70-80 \mathrm{~cm}$. Fr blanco y café. Ene. SBC. ASM 654, 669.

Brickellia filipes B.L.Rob. Hierba $50 \mathrm{~cm}$. Fl blanca. Feb. SBC. ASM 1222.

Chromolaena odorata (L.) R.M.King et H.Rob. Arbusto 80 cm-2.5 m. Fl blanca. Nov.-dic. SBC, Ga. ANZ 673, 795, 912; ASM 475, 602 .

Critoniopsis triflosculosa (Kunth) H.Rob. Árbol o arbusto 60 cm. Fl blanca. Feb. SBC. SAY 5444; KV 684.

Dyssodia aurantia (L.) B.L.Rob. Hierba 1-2.5 m. Fl roja o anaranjada. Feb.-may. SBC. ASM 830, 1309, 1415.

Eclipta prostrata (L.) L. Hierba $60 \mathrm{~cm}$. Fr verde. Dic. SBC. ANZ 681, 1375; SS 5410; SAY 4173, 4367.

Espejoa mexicana DC. Hierba 40 cm. Fl roja. Sept. SAY 4230.

Lagascea decipiens Hemsl. Hierba $60 \mathrm{~cm}$. Fr verde. Dic. SBC. ASM 1262.

Melampodium divaricatum (Rich.) DC. Hierba $60 \mathrm{~cm}$. FI amarilla. Sept. SMS. SAY 4192.

Melampodium linearilobum DC. Hierba 20-30 cm. Fl amariIla. Sept. Sa, SBC2. ME 7278; SAY 4226.

Melampodium nutans Stuessy. Hierba $15-30 \mathrm{~cm}$. FI amarilla. Sept.-oct. SBC. SS 5383; SAY 4266, 5237.

Melanthera aspera (Jacq.) Small. Hierba 20 cm-1.5 m. Fl blanca. Oct.-nov. SBC. ANZ 576; ASM 404; SS 5420; SAY 5124.

Melanthera nivea (L.) Small. Hierba $60-80 \mathrm{~cm}$. Fl blanca. Dic.-feb. SBC. ANZ 701, 794, 866; SAY 5298, 5453.

Milleria quinqueflora L. Hierba $50 \mathrm{~cm}-1.5 \mathrm{~m}$. Fl amarilla. Sept.-oct. SBC. ME 7339; SAY 5087.
Otopappus epaleaceus Hemsl. Bejuco. Fr seco. Feb. SBC. SAY 5422.

Otopappus microcephalus S.F.Blake. Árbol $8 \mathrm{~m}$. Fl verde. Oct. SBC. ASM 228.

Pluchea carolinensis (Jacq.) G.Don. Arbusto 1.5-1.8 m. FI rosa. Feb. SBC. ANZ 963; ASM 650; SAY 5282, 5177.

Pluchea symphytifolia (Mill.) Gillis. Arbusto $2 \mathrm{~m}$. Fl morada. May. SBC. ANZ 1039; KV 723.

Porophyllum macrocephalum DC. Hierba $50 \mathrm{~cm}-1 \mathrm{~m}$. FI verde. Oct. SBC. ANZ 540, 707; ASM 306, 520; SAY 5093.

Porophyllum punctatum (Mill.) S.F.Blake. Hierba 1-2 m. FI rojiza. Ene. SBC. ANZ 827; ASM 595; SAY 4301.

Porophyllum viridiflorum (Kunth) DC. Hierba $80 \mathrm{~cm}$. Fr café. Nov. SBC. ASM 498.

Sinclairia sublobata (B.L.Rob.) Rydb. Bejuco. Infl. inmadura, látex. Nov. SBC. GJ 1873.

Tehuana calzadae Panero et Villaseñor. Hierba 30-60 cm. FI anaranjada o amarilla. Sept.-oct. SBC. ASM 26; SAY 4261, 5022, 5232, 5236 .

Tithonia rotundifolia (Mill.) S.F.Blake. Arbusto $80 \mathrm{~cm}-2.5 \mathrm{~m}$. Fl amarilla o anaranjada. Oct-ene. SBC. ANZ 495, 536, 669; ASM 202, 488.

Tithonia tubiformis (Jacq.) Cass. Arbusto $2.5 \mathrm{~m}$. Fl amarilla. Nov. SBC. ASM 390

Tridax procumbens L. Hierba $40 \mathrm{~cm}$. Fl blanca. Dic-ene. SBC. ANZ 724, 877; ASM 1314, 1315; SAY 4291.

Tridax tenuifolia Rose. Hierba 25 cm-1 m. Fl blanca. Sept.oct. SBC, SBCE. ANZ 490; ASM 117; SAY 4462, 5018.

Trixis hintoniorum B.L.Turner. Arbusto $1.5 \mathrm{~m}$. Fl amarilla. Oct. SBC. ANZ 459.

Trixis inula Crantz. Hierba 80 cm. Fl amarilla. Dic. SBC. ANZ 784.

Trixis michuacana Lex. Arbusto $80 \mathrm{~cm}-1.5 \mathrm{~m}$. Fl amarilla. Oct. SBC. ANZ 459, 493.

Trixis silvatica B.L.Rob. et Greenm. Arbusto $80 \mathrm{~cm}-3 \mathrm{~m}$. FI blanca o amarilla. Ene.-feb. SBC, VD, Ma, Ga. ANZ 828, 973, 985; ASM 567, 587.

Trixis sp. Bejuco. SBC. ASM 1626.

Verbesina platyptera Sch.Bip. ex Klatt. Arbusto 2-2.5 m. FI verde. Oct.-nov. SBC. ASM 373; SAY 5123, 5153.

Viguiera gracillima Brandegee. Hierba $60 \mathrm{~cm}-1 \mathrm{~m}$. Fl amariIla. Oct.-nov. SBC. ANZ 580; SAY 5229. 
Wedelia tehuantepecana B.L.Turner. Hierba 80 cm-1.5 m. Fl amarilla. Oct. SBC. ASM 271, 313.

Zinnia peruviana (L.) L. Hierba 30-40 cm. Fl verde. Oct. SBC. ASM 69, 96; SAY 4269.

Zinnia zinnioides (Kunth) Olorode et Torres. Hierba $25 \mathrm{~cm}$. Fr verde. Oct. SBC. ASM 60.

\section{BATACEAE}

Batis maritima L. Hierba rastrera $50 \mathrm{~cm}$. Fl blanca. Mar.-abr. SBC, SMS. ANZ 1333, 1337; ASM 700.

\section{BIGNONIACEAE}

Amphilophium paniculatum (L.) Kunth. Bejuco. Fl rosa. Oct. SBC. ASM 1154.

Arrabidaea floribunda (Kunth) Loes. Bejuco. Fr seco. Dic. SBC. ANZ 762.

Arrabidaea mollissima (Kunth) Bureau et K.Schum. Bejuco. FI rosa, morada o lila. Ene.-abr. SBC. ANZ 1008, 844, 958; ASM 1261; KV 696.

Astianthus viminalis (Kunth) Baill. Árbol 4-8 m. FI amarilla. Mar.-jun. Ga. ANZ 1265; ASM 1128, 1146, 403; SAY 5506.

Crescentia cujete L. Árbol 4 m. Fr verde. Oct. SBC. ANZ 432.

Cydista diversifolia (Kunth) Miers. Bejuco. Fl rosa, morada o lila. Ago.-oct. SBC, SMS. AÁH 51; ME 7052, 7356; GJ 1950; ANZ 1161.

Mansoa hymenaea (DC.) A.H.Gentry. Bejuco. FI rosa, olor a ajo. Mar. SBC. ME 6445.

Paragonia pyramidata (Rich.) Bureau. Bejuco. Fl rosa. Mar.abr. SBC. ME 6979; GJ 2035; ANZ 1364.

Parmentiera aculeata (Kunth) Seem. Árbol 8 m. Fl verde. Feb. SBC. ASM 1241.

Pithecoctenium crucigerum (L.) A.H.Gentry. Bejuco. Fl blanca. Jul. SBC. ASM 596; SAY 5542, 5620.

Tabebuia chrysantha (Jacq.) G.Nicholson. Árbol 3 m. Fl amarilla. Ene.-feb. SBC. GJ 1746.

Tabebuia donnell-smithii Rose. Árbol 8 m. Fr seco. Sept. SBC. ANZ 1224.

Tabebuia impetiginosa (Mart. ex DC.) Standl. Árbol 8-12 m. FI rosa. Dic.-ene. SBC. GJ 1902; SS 3796, 5455.

Tabebuia rosea (Bertol.) A.DC. Árbol 7-18 m. Fl rosa. Feb.may. SBC. GJ 2034; ASM 1296, 1379, 811.

Tecoma stans (L.) Juss. ex Kunth. Árbol o arbusto 2.5-6 m. Fl amarilla. Nov.-abril. SBC. ANZ 1026, 505; ASM 1242, 499.
Xylophragma seemannianum (Kuntze) Sandwith. Bejuco. FI lila. May. SMS. GJ 2245.

\section{BIXACEAE}

Cochlospermum vitifolium (Willd.) Spreng. Árbol 3-7 m. Fl amarilla. Dic.-ene. SBC. GJ 1915; ANZ 781, 845, 962; ASM 548.

\section{BOMBACACEAE}

Ceiba aesculifolia (Kunth) Britten et Baker f. Árbol 7-12 m. FI blanca. Feb. SBC. GJ 2372; SS 5571; ASM 1295.

Ceiba parvifolia Rose. Árbol 6 m. FI verde. Feb. SBC. SAY 5389.

Ceiba pentandra (L.) Gaertn. Árbol 8 m. SBC. ASM 1220.

Pseudobombax ellipticum (Kunth) Dugand. Árbol 4.5 m. Fr café. Abr. SBC. ANZ 1019.

\section{BORAGINACEAE}

Cordia alliodora (Ruiz et Pav.) Oken. Árbol 3-13 m. Fl blanca. Feb. SBC. GJ 1835; ASM 1216, 438, 453,495.

Cordia curassavica (Jacq.) Roem. et Schult. Arbusto 2-2.5 m. Fl blanca o amarilla. Jul.-ago. SBC, Sa. ME 7038; SAY 4239, 5656.

Cordia dentata Poir. Arbusto 2-8 m. Fl amarilla. Ago.-dic. SBC, SBCE, SMS. AÁH 53, 75, 82; ANZ 1258, 541.

Cordia elaeagnoides DC. Árbol 4-10 m. Fl blanca. Jul.-dic. SBC. ANZ 1177, 527, 670; ASM 1138, 222.

Cordia inermis (Mill.) I.M.Johnst. Arbusto 60 cm-1 m. Fl blanca. Ago.-oct. SBC. ANZ 1095, 1135; ASM 41.

Cordia oaxacana DC. Arbusto 1-2 m. Fl blanca. Ago.-sept. SBC. ME 7041; ANZ 1225; SS 5454.

Cordia seleriana Fernald. Árbol o arbusto 2-10 m. Fl blanca. Abr.-ago. SBC. GJ 1858, 1886, 1908; ANZ 1513; ASM 1000.

Cordia tinifolia Willd. ex Roem. et Schult. Árbol 4.5-13 m. SBC. GJ 1772, 1923.

Cordia truncatifolia Bartlett. Árbol o arbusto 1-9 m. Fl blanca. Jun. SBC. ANZ 1525; ASM 1599; SAY 5495.

‡ Ehretia tinifolia L. Árbol $4 \mathrm{~m}$. Fl amarilla. Sept. SBC. ANZ 1223.

Heliotropium angiospermum Murray. Hierba 30-60 cm. FI blanca. Todo el año. SBC. ME 7347; ANZ 438, 563, 899; ASM 1304.

Heliotropium curassavicum L. Hierba rastrera hasta $20 \mathrm{~cm}$. FI blanca. Mar.-abr. Pz. ANZ 1371; ASM 1348.

Heliotropium fruticosum L. Hierba $3 \mathrm{~cm}$. Fl blanca. Ago. SBC. ASM 978. 
Heliotropium indicum L. Hierba $50 \mathrm{~cm}$. Fl blanca. Jun. SBC. G) 2378.

Heliotropium procumbens Mill. Hierba rastrera $60 \mathrm{~cm}$. FI blanca. Jun.-oct. SBC, SMS. ME 7355; GJ 2381, 2392; ANZ 1259; ASM 1399.

Heliotropium ternatum Vahl. Hierba 20-30 cm. Fl blanca. May.-jul. SBC, SBC2. ASM 823; SAY 5727.

Tournefortia calycina Benth. Arbusto 30 cm-1.5 m. Fl blanca. Oct.-mar. SBC, SBCE. ANZ 477; SS 5499; ASM 1351, 1354, 334.

Tournefortia densiflora M.Martens et Galeotti. Arbusto 80 cm-2 m. Fl blanca. Dic.-feb. SBC, VD, Ma. ANZ 803, 804, 940b; ASM 681; SAY 5384.

Tournefortia hirsutissima L. var. bicolor (Sw.) D.L.Nash. Arbusto escandente 2.5 m. Fl blanca. Abr. SBC. ASM 1428, 1449.

Tournefortia volubilis L. Arbusto $2 \mathrm{~m}$. Fr verde. Ago.-feb. SBC, VD, Ma. ME 7049; SAY 4476, 5367.

\section{BURSERACEAE}

Bursera arborea (Rose) L.Riley. Árbol 4-14 m. Fr verde. Ago.oct. SBC. GJ 1806; ANZ 1054, 1170; ASM 1075, 1215.

Bursera aff. cinerea Engl. Árbol 4-7 m. Fr verde. Sept.-oct. SBC, SBCE. ASM 138; SAY 4440.

Bursera excelsa (Kunth) Engl. Árbol o arbusto 1-13 m. FI verde-amarillenta. May.-oct. SBC, VD, Ma. AÁH 57; GJ 1839; ANZ 1182, 1210, 1506.

Bursera aff. fagaroides (Kunth) Engl. Árbol 4-5 m. Fr verde. Sept.-oct. SBC, Sa. ASM 101, 48; SAY 4236.

Bursera heteresthes Bullock. Árbol o arbusto 2-10 m. Fr verde. Jul.-oct. SBC. ME 7051; SS 5388; ASM 1049; SAY 5580, 5648.

Bursera instabilis McVaugh et Rzed. Árbol o arbusto 1.5-2 m. FI amarilla. May. SBC. ASM 756; SAY 4463.

Bursera krusei Rzed. Árbol 2-10 m. Fr verde. Jun.-oct. SBC. G) 1801; ANZ 1117, 814; SS 5377, 5422.

Bursera laurihuertae Rzed. et Calderón. Árbol 4.5-6 m. Fr verde. Oct.-dic. SBC. ANZ 498; ASM 122, 154, 169, 544.

Bursera aff. longicuspis Lundell. Árbol 10-12 m. Fr verde. Jul. SBC. SAY 5632, 5661.

Bursera sarcopoda Paul G.Wilson. Árbol 7-14 m. Fr verde. Jul.-ago. SBC. GJ 2386; ANZ 1048; ASM 1024, 913; SAY 5624.

Bursera schlechtendalii Engl. Árbol 3-5 m. Fr verde o café. Sept.-oct. SBC, SBCE. ASM 123; SAY 4441.
Bursera simaruba (L.) Sarg. Árbol 4-14 m. Fr verde. Ago.-dic. SBC, SMS. GJ 1822; ANZ 1178, 699, 749; ASM 1001.

Bursera sp. Árbol o arbusto 2-7 m. Fr verde o café. Ene.-feb. SBC. ASM 573; SAY 5418, 5429.

Bursera sp. nov. Árbol 3 m. Fl blanca, corteza verde. Jun. SBC. GJ 1747, 1794; ANZ 1020, 840; ASM 505.

\section{CACTACEAE}

Opuntia decumbens Salm-Dyck. Arbusto rastrero $1 \mathrm{~m}$. FI anaranjada. Dic. SBC. ANZ 798; ASM 795; SAY 5481.

Opuntia dejecta (Salm-Dyck) Salm-Dyck. Arbusto 1.5-4 m. FI roja o rosa mexicano. Oct.-jun. SBC, Ma, VD, SMS. ASM 300, 440, 558; ANZ 836; SAY 5373.

Opuntia tehuantepecana (Bravo) Bravo. Arbusto $3 \mathrm{~m}$. Fr verde. Sept. SBCE. SAY 4383.

Opuntia velutina F.A.C.Weber. Arbusto decumbente. FI amarilla. Dic. SBC. SS 5502.

Peniocereus oaxacensis (Britton et Rose) D.R.Hunt. Arbusto $60 \mathrm{~cm}$. Fr verde o rojo. Feb.-ago. SBC. SAY 4274, 4305, 5480 .

Pereskia lychnidiflora DC. Árbol 4-10 m. Fl roja. Ago. SBC. ANZ 1072, 1286; ASM 551; SAY 5255.

Pereskiopsis diguetii (F.A.C.Weber) Britton et Rose. Arbusto suculento. SBC. ANZ 479.

Pereskiopsis kellermanii Rose. Arbusto $60 \mathrm{~cm}$. SBCE. SAY 4466.

Pereskiopsis rotundifolia (DC.) Britton et Rose. Arbusto ramificado $80 \mathrm{~cm}$. SBC. SAY 5672.

Stenocereus chacalapensis (Bravo et T.MacDoug.) Buxb. Árbol candelabriforme $5 \mathrm{~m}$. FI rojiza. Feb. SBC, SMS, Ma, VD. ANZ 469; SAY 4360.

\section{CAPPARACEAE}

Capparis flexuosa (L.) L. Árbol, arbusto escandente 2-3 m. Fl blanca o amarilla. Feb.-jun. SBC, SBCE. ANZ 1144, 1521; SS 5554; ASM 1030, 783.

Capparis frondosa Jacq. Árbol o arbusto $50 \mathrm{~cm}-3 \mathrm{~m}$. Fl blanca. Oct.-abr. SBC. ANZ 1004, 1145, 1365, 439.

Capparis incana Kunth. Árbol o arbusto 1.5-6 m. Fl blanca. Mar.-may. SBC. GJ 1906, 1910, 2041; ANZ 1321; ASM 767.

Capparis indica (L.) Druce. Árbol o arbusto 1-9 m. Fl blanca o rosa. Mar.-jun. SBC, SBCE. GJ 1830; ANZ 1352; SS 5612, 5632; ASM 1358.

Capparis odoratissima Jacq. Árbol o arbusto 3-6 m. Fl verdes o lilas. Mar.-may. SBC, SMS. ASM 699, 728; SAY 4371; KV 734. 
Capparis verrucosa Jacq. Árbol o arbusto 1-5 m. Fl blanca. Feb.-may. SBC, Pz. GJ 1921, 2042, 2051; ANZ 1040, 1330.

Cleome aculeata L. Hierba 25-80 cm. jul.-oct. SBC. ANZ 1097b; ASM 1051, 193, 312; SAY 5585.

Cleome gynandra L. Hierba $80 \mathrm{~cm}$. Fl blanca. Sept. SBC. ANZ 1227.

Cleome spinosa Jacq. Hierba $70 \mathrm{~cm}$. Fl blanco-rosa. Oct. SBC. ASM 1101.

Cleome sp. Hierba $60 \mathrm{~cm}$. Fr secos. Ene. SBC. ANZ 934.

Crateva tapia L. Árbol 3-15 m. Fl blanca. Mar.-may. SBC, Ga. ANZ 1002, 1014, 1319; ASM 1155, 1369.

Forchhammeria pallida Liebm. Árbol 3-7 m. Fl blanca. Nov. SBC, VD, Ma. SS 5565; ASM 1244, 433, 457; SAY 4286.

Morisonia americana L. Árbol o arbusto 1.5-7 m. Fl blanca. Oct.-feb. SBC, Ga. GJ 1815, 2040; ANZ 1005, 462; SS 5457.

Polanisia viscosa (L.) DC. Hierba $20 \mathrm{~cm}-1 \mathrm{~m}$. Fl amarilla. Ago.-oct. SBC, SMS. AÁH 60; ANZ 1238, 1278; ASM 279, 384.

\section{CARICACEAE}

‡ Carica papaya L. Árbol 3-10 m. Fl blanca amarillenta. Ago.nov. SBC, SMS. AÁH 72; ANZ 572; ASM 277; SAY 4218.

Jacaratia mexicana A.DC. Árbol 4-8 m. Fl blancas o amariIlas. Nov.-feb. SBC. GJ 1899; ANZ 1289, 849; 977; ASM 557.

Jarilla chocola Standl. Hierba 10-40 cm. Fl blanca con rosa o crema. May. SBC. GJ 2200, 2224; ANZ 1068; SS 5929; ASM 1461.

\section{CELASTRACEAE}

Crossopetalum uragoga (Jacq.) Kuntze. Arbusto $40 \mathrm{~cm}-1.5 \mathrm{~m}$. FI roja o guinda. Jun.-ene. SBC, SBCE, VD, Ma. AÁH 59; ANZ 468, 790; SS 3802, 5484.

Wimmeria persicifolia Radlk. Arbusto 1.5-3 m. Fr secos samaroides. Oct.-ene. SBC, VD. ANZ 458.

\section{CHRYSOBALANACEAE}

Licania arborea Seem. Árbol 3-15 m. FI amarilla. Ene. SBC. ANZ 950; ASM 1235, 570; SAY 5304; KV 659.

\section{COMBRETACEAE}

Bucida macrostachya Standl. Árbol 5-9 m. Fl blanca o amarilla. Jul.-oct. SBC. ASM 1324, 170; SAY 5649.

Combretum decandrum Jacq. Bejuco. Fl blanca. Ene. SBC. ANZ 1519; SAY 5364.

Combretum fruticosum (Loefl.) Stuntz. Bejuco o arbusto escandente 1.8-3 m. Fl roja. Oct.-mar. SBC, SBC2, Ma, SMS. G) 1938, 2032; ANZ 1149, 1347; ASM 141.
Combretum laxum Jacq. Bejuco. Fl con estambres exertos naranjas. Feb. SBC. ASM 1219.

Conocarpus erectus L. Árbol 3-5 m. Fr verde o café. Feb.sept. VD, Ma, SBC. ANZ 1206, 989; SAY 4485, 5370, 5543.

Laguncularia racemosa (L.) C.F.Gaertn. Árbol 4 m. Fl blanca verdosa. May.-sept. SBC, SBCE. ANZ 1032; SAY 4471, 4486.

\section{CONNARACEAE}

Rourea glabra Kunth. Bejuco. Fl blanca o crema. Sept. SBC, SMS. GJ 2030; ANZ 1041; SS 5474, 5540; ASM 1087.

\section{CONVOLVULACEAE}

Aniseia martinicensis (Jacq.) Choisy. Bejuco o hierba rastrera. FI blanca. Sept.-mar. SMS, Ga, Pz. ASM 1342; SAY 4373; 4439.

Evolvulus alsinoides (L.) L. Hierba $40 \mathrm{~cm}$. Fl morada. Oct. SBC. ASM 1118.

Evolvulus cardiophyllus Schltdl. Hierba $40 \mathrm{~cm}$. FI morada. Oct. SBC. ASM 231.

Ipomoea aff. alba L. Bejuco. Fr verde. Nov. SBC. ASM 483.

Ipomoea bracteata Cav. Bejuco. Fl morada o guinda. Dic.mar. SBC, Ga. GJ 1960; ANZ 971, 983; SS 5518; ASM 612.

Ipomoea dumosa (Benth.) L.O.Williams. Bejuco. Fl morada. Oct. SBC. ASM 1175.

Ipomoea gesnerioides J.A.McDonald. Bejuco. Fl blanco-amarillenta con tubo negro en el interior. Dic. SBC. ANZ 780.

Ipomoea hederifolia L. Bejuco. FI naranja o roja. Ago.-feb. SBC. ANZ 1123, 531; SS 5400; ASM 1123, 414.

Ipomoea meyeri (Spreng.) G.Don. Bejuco. FI morada con blanco. Oct.-dic. SBC. SS 5541; ASM 1203, 478.

Ipomoea microsepala Benth. Bejuco. FI amarilla. Nov.-feb. SBC. ANZ 1317, 688, 925; SS 5560; SAY 5284.

Ipomoea minutiflora (M.Martens et Galeotti) House. Bejuco. FI amarilla o salmón. Oct.-nov. SBC. SS 5436; ASM 357; SAY 5208.

Ipomoea nil (L.) Roth. Bejuco. FI morada o guinda con blanco. Oct.-feb. SBC. ANZ 513, 561; SS 5429; ASM 1141, 1209.

Ipomoea pedicellaris Benth. Bejuco. Fl morada. Oct.-feb. SBC. ANZ 684, 935; SS 5564; ASM 200.

Ipomoea pes-caprae (L.) R.Br. Bejuco. FI morada. Dic. SBC, SBCE. SS 5487; SAY 4442, 4467.

Ipomoea populina House. Bejuco. Fl blanca con interior de tubo vino obscuro. Dic.-ene. SBC. ANZ 825; SS 5453, 5504. 
Ipomoea praecana House. Bejuco. Fr café, látex. Mar.-abr. SBC. GJ 1848, 1945; ANZ 1324; ASM 1327.

Ipomoea quamoclit L. Bejuco. FI rojo intenso o rosa mexicano. Sept.-dic. SBC. ANZ 1179; SS 5542; ASM 226; SAY 4411.

Ipomoea robinsonii House. Bejuco. FI blanca. Sept. SBC. ASM 1071.

Ipomoea setosa Ker Gawl. Bejuco. Fl morada. Sept.-dic. SBC. SS 5531; ASM 1079.

Ipomoea ternifolia Cav. var. ternifolia. Bejuco. FI morada o rosa mexicano. Ago.-ene. SBC, SBC2, Sa. ASM 572; SAY 4232.

Ipomoea tiliacea (Willd.) Choisy. Hierba rastrera. FI morada. Nov. SBC. ANZ 543.

Ipomoea trifida (Kunth) G.Don. Bejuco. FI rosa o morada. Oct.-feb. SBC. ANZ 702, 772, 858; SS 5553; ASM 1174.

Ipomoea triloba L. Bejuco. FI morada. Oct.-nov. SBC. ANZ 514; ASM 1189, 1201, 355, 474.

Ipomoea umbraticola House. Bejuco. Fl lila de gran tamaño. Oct. SBC. ASM 1187.

Jacquemontia agrestis (Mart. ex Choisy) Meisn. Bejuco. FI morada. Oct. SBC. ASM 34.

Jacquemontia nodiflora (Desr.) G.Don. Bejuco. FI blanca. Oct. SBC. ANZ 734; SS 5465; ASM 190, 471, 51.

Jacquemontia oaxacana (Meisn.) Hallier f. Bejuco. Fr verde y café. Ene. SBC. ASM 680.

Jacquemontia pentantha (Jacq.) G.Don. Bejuco. FI blanca. Oct. SBC. SS 5430.

Jacquemontia polyantha (Schltdl. et Cham.) Hallier f. Bejuco. FI blanca o lila. Oct.-feb. SBC. ANZ 484, 881; SS 5561; ASM 113.

Jacquemontia pringlei A.Gray. Bejuco. Fr verde. Dic.-ene. SBC. ASM 534, 640.

Merremia aegyptia (L.) Urb. Bejuco. Fl blanca. Oct.-dic. SBC. ANZ 544, 773; SS 5416, 5442; ASM 209.

Merremia cissoides (Lam.) Hallier f. Hierba rastrera. Fl blanca. Dic. SBC. ANZ 700.

Merremia dissecta (Jacq.) Hallier f. Bejuco. Fr seco. Dic. SBC. SS 5512.

Merremia quinquefolia (L.) Hallier f. Bejuco o hierba rastrera. FI blanca o amarilla. Oct.-may. SBC, Ma. ANZ 1345, 515, 521; SS 5479; ASM 1372.
Merremia umbellata (L.) Hallier f. Bejuco. Fl amarilla. Ene.feb. SBC. ANZ 859; SAY 5318, 5319; KV 665.

Turbina corymbosa (L.) Raf. Bejuco. Fl blanca. Ene.-feb. SBC. ANZ 919; SAY 5452.

\section{CUCURBITACEAE}

Cayaponia attenuata (Hook. et Arn.) Cogn. Bejuco. Fr verde. Ene.-abr. SBC. ANZ 933; ASM 1375; SAY 5283.

Chalema synanthera Dieterle. Bejuco. FI blanca. Sept.-dic. SBC. ANZ 586; SS 5424, 5446; SAY 4281, 4336.

‡ Citrullus lanatus (Thunb.) Matsum. et Nakai. Bejuco. FI amarilla. Ago. SBC. ASM 1064.

Cucumis anguria L. Bejuco. Fr blanco. Ene. SBC. ANZ 923.

Cucurbita argyrosperma K.Koch. Bejuco. FI amarilla. Sept.nov. SBC. ASM 413; SAY 4320.

Cucurbita pepo L. Bejuco. Fl amarilla. Ago. SBC. AÁH 69.

Cyclanthera multifoliola Cogn. Bejuco. FI blanca o verde. Sept.-nov. SBC. SS 5417; ASM 1082, 411; SAY 5083.

Dieterlea sp. Bejuco. Fr amarillo o anaranjado. Ago.-sept. SBC. ANZ 1151, 1217.

Doyerea emetocathartica Grossourdy. Bejuco. Fl café. Abr. SBC. ANZ 1013; SS 5921.

Echinopepon racemosus (Steud.) C.Jeffrey. Bejuco. FI blanca. Nov. SBC. ANZ 908; ASM 323.

Ibervillea hypoleuca (Standl.) C.Jeffrey. Bejuco. Fr verde con blanco. Jul. SBC. SAY 5596.

Melothria pendula L. Bejuco. FI amarilla. Ago.-nov. SBC. ANZ 1070, 1119; ASM 1083, 235; SAY 5157.

Momordica charantia L. Bejuco. FI amarilla. Abr.-dic. SBC, Ga. ANZ 1130, 1241; SS 5498; ASM 1113, 1125.

Polyclathra cucumerina Bertol. Bejuco. Fl blanca. Nov. SBC. ANZ 518.

Rytidostylis gracilis Hook. et Arn. Bejuco. FI amarilla o verdosa. Sept.-oct. SBC. ANZ 1176, 584; SS 5389; ASM 1074, 1085.

Schizocarpum palmeri Cogn. et Rose. Bejuco. FI amarilla. Sept.-oct. SBC. ANZ 1181; ASM 118, 281.

Sicydium schiedeanum Schltdl. Bejuco. Fl blanca. Ago. SBC. ANZ 1064.

DILLENIACEAE

Curatella americana L. Árbol 6-7 m. Fl blanca. Ago.-feb. SBC, Sa. ASM 1273, 964. 


\section{EBENACEAE}

Diospyros aequoris Standl. Árbol o arbusto 2-8.5 m. Fl blanca o amarilla. Jun.-sept. SBC, Sa, VD. AÁH 62; GJ 1911, 1922; ANZ 461, 805.

Diospyros salicifolia Humb. et Bonpl. ex Willd. Arbusto 2.54 m. Fr verde. Oct.-dic. SBC. ANZ 506, 742; ASM 530.

\section{ERYTHROXYLACEAE}

Erythroxylum havanense Jacq. Árbol o arbusto 2-7 m. Fl blanca. May. SBC. GJ 1787, 1894; ASM 779, 928; SAY 4270.

\section{EUPHORBIACEAE}

Acalypha alopecuroidea Jacq. Hierba $30 \mathrm{~cm}$. Fr verde. Ago. SBC. ANZ 1084, 1088.

Acalypha havanensis Müll.Arg. Hierba $60 \mathrm{~cm}$. Fl blanca. Ago. SBC. AÁH 71.

Acalypha microphylla KI. Hierba $30 \mathrm{~cm}$. FI morada. May. SBC. SS 5900.

Acalypha sp. Hierba 60 cm. Fl verde. Oct. SBC. ASM 90.

Astrocasia sp. Árbol o arbusto 1.5-3 m. Fl blanca. Oct.-nov. SBC. ANZ 565; ASM 237; SAY 5245.

Bernardia mexicana (Hook. et Arn.) Müll.Arg. Arbusto 3 m. Botones. Dic. SBC. SS 5533.

Cnidoscolus multilobus (Pax) I.M.Johnst. Hierba $40 \mathrm{~cm}-2 \mathrm{~m}$. Fl blanca. Todo el año. SBC, SBC2, Ga. ANZ 1264; ASM 1108, 125; SAY 4288, 5090.

Cnidoscolus tubulosus (Müll.Arg.) I.M.Johnst. Árbol o arbusto 2-6 m. Fl blanca. Abr.-sept. SBC, SBC2, SBCE, Ma. ANZ 1031, 1349; ASM 769, 790; SAY 4300.

Croton alamosanus Rose. Arbusto 2 m. Botones. Dic. SBC. ASM 528.

Croton axillaris Müll.Arg. Árbol 4 m. Botones. Dic. SBC. GJ 1914.

Croton flavescens Greenm. Arbusto 2.5 m. Fr verde. Ago. SBC. ASM 1035.

Croton lobatus L. Hierba 40-70 cm. Fr verde. Jul.-sept. SBC. ANZ 1045, 1251; SAY 5746.

Croton mazapensis Lundell. Árbol 3 m. Fr verde. Oct. SBC. SAY 5246

Croton niveus Jacq. Árbol o arbusto 50 cm-6 m. Fl blanca. Ago.-oct. SBC, SBC2. GJ 1795, 1813; ANZ 1311, 984; SS 5379 .

Croton pseudoniveus Lundell. Árbol 5 m. Fr café. Nov. SBC. ASM 326.
Croton septemnervius McVaugh. Árbol 5 m. SBC. GJ 1913.

Croton suberosus Kunth. Arbusto 1-2.5 m. Fl blanca amariIlenta o verde, corteza corchosa. Ago.-feb. SBC, SBCE, Sa. ANZ 1010, 1050; SS 5581; ASM 1016, 38.

Croton yucatanensis Lundell. Arbusto 2.5 m. Fr verde. Ago. SBC. ASM 935.

Croton sp. Árbol o arbusto 1-3 m. Fr verde. Oct. SBC. SAY 5106, 5258a, 5270.

Dalechampia scandens L. Hierba rastrera o bejuco. FI blanca, amarilla o verde. May.-dic. SBC, SBC2. ANZ 1152, 737; SS 5396, 5527; ASM 160.

Ditaxis guatemalensis Pax et K.Hoffm. Hierba 30-60 cm. FI blanca. May.-oct. SBC, SBC2. ASM 1252, 268, 824.

Ditaxis manzanilloana (Rose) Pax et K.Hoffm. Hierba 40-60 cm. Fl blanca. Ene. SBC, Ga. ASM 623; SAY 5417.

Ditaxis sp. Hierba 50 cm. Fl blanca. Dic. SBC. ANZ 800.

Euphorbia aff. delicatula Boiss. Arbusto 1.5-2 m. Fl verde. Nov.-ene. Látex. SBC. ASM 421; KV 678.

Euphorbia francoana Boiss. Hierba $1.4 \mathrm{~m}$. Fl morada. Oct. SBC. ASM 1172.

Euphorbia graminea Jacq. Hierba $60 \mathrm{~cm}$. Fr verde. Oct. SBC. SAY 5015.

Euphorbia hirta L. Hierba sufrutescente $10-80 \mathrm{~cm}$. FI verde o blanca. Oct. SBC. ASM 272, 311, 452; SAY 5062.

Euphorbia hyssopifolia L. Hierba $80 \mathrm{~cm}-1 \mathrm{~m}$. Fl blanca. Dic. SBC. ANZ 685, 761.

Euphorbia lasiocarpa Klotzsch. Hierba $20 \mathrm{~cm}$. FI amarilla. Dic. SBC. ANZ 759.

Euphorbia schlechtendalii Boiss. Arbusto 1-3 m. Fr verde. Dic. SBC. G) 1818; ANZ 748.

Euphorbia segoviensis (Klotzsch et Garcke) Boiss. Arbusto 60 cm-1.5 m. Fl blanca. Sept.-nov. SBC. ANZ 585; ASM 128, 162; SAY 5012, 5135.

Euphorbia thymifolia L. Hierba rastrera. FI blanca. Jul. SBC. SAY 5710.

Hippomane mancinella L. Árbol o arbusto 1.5-14 m. Feb.abr. SBC, SMS. AÁH 97; ANZ 1141, 993; SS 5577; ASM 695.

Hura polyandra Baill. Árbol 15 m. Fr verde. Feb. SBC. ASM 1239.

Jatropha curcas L. Arbusto $2 \mathrm{~m}$. Fr verde. Oct. SBC. ASM 1098, 1145. 
Jatropha malacophylla Standl. Árbol 5-6 m. Fl blanca. Jun. SBC. SAY 5540, 5612.

Jatropha sympetala S.F.Blake et Standl. Árbol 3-5 m. FI guinda. Jun. SBC, SBCE. ASM 1050; SAY 4451, 5549.

Manihot chlorosticta Standl. et Goldman. Arbusto 3 m. SBC. ANZ 517.

Manihot oaxacana D.J.Rogers et Appan. Arbusto escandente o bejuco. Fl verde. May-nov. SBC, SMS. AÁH 87; GJ 1796 , 2223; ANZ 1057, 1115.

Phyllanthus elsiae Urb. Árbol o arbusto 2.5-12 m. Fl rosa. Mar. Pz, SBC. ANZ 997; ASM 1336, 1337, 1339, 1482.

Phyllanthus galeottianus Baill. Arbusto $3 \mathrm{~m}$. Botones. Jul. SBC. SAY 5640.

Phyllanthus mocinianus Baill. Árbol o arbusto 1.5-5 m. FI blanca. Jun. SBC. ANZ 1307; SS 5423, 5525; SAY 5498, 5625.

‡ Ricinus communis L. Arbusto $70 \mathrm{~cm}-2 \mathrm{~m}$. Fl verde. Ene. SBC. ANZ 709, 863.

Sapium macrocarpum Müll.Arg. Árbol 6-14 m. Fl amarilla o verde. Jun. SBC, SMS. GJ 2375; ANZ 1071, 1096; ASM 1004; SAY 4353.

Sebastiania pavoniana (Müll.Arg.) Müll.Arg. Árbol 8 m. SBC. G) 1926.

Tragia glanduligera Pax et K.Hoffm. Hierba rastrera o bejuco. Fl blanca, urticante. Dic. SBC. SS 5460; ASM 289, 309, 512.

\section{FLACOURTIACEAE}

Casearia corymbosa Kunth. Árbol o arbusto 1.5-6 m. Fl blanca o amarilla. May.-jun. SBC, Ma. AÁH 88, 90; GJ 2181, 2185; ANZ 1147.

Casearia tremula (Griseb.) Griseb. ex C.Wright. Árbol 4-8 m. Botones. May. SBC. GJ 1736, 1903; ASM 1815; SAY 5576, 5659.

Homalium trichostemon S.F.Blake. Árbol 5-15 m. Fl amarilla o blanca. Feb.-oct. SBC, SBC2, SMS. ANZ 1128, 1139; ASM 1377, 719; SAY 4219.

Muntingia calabura L. Árbol o arbusto 1-8 m. Fl blanca. Ago.feb. SBC, SBC2, SMS. AÁH 96; ANZ 1097a, 671; ASM 807; SAY 4329.

Prockia crucis P.Browne ex L. Árbol 2.5-3.3 m. Fr verde. Jul. SBC. GJ 1771, 2684; ASM 1821.

Samyda mexicana Rose. Árbol 3-8 m. Fl blanco verdosa. May.-ago. SBC. GJ 2234; SS 5574, 5924; ASM 1012, 1056.

Xylosma flexuosa (Kunth) Hemsl. Árbol 7-8 m. Fl amarilla. Oct. SBC, Sa. SS 5539; ASM 1263, 252, 461, 554.
Xylosma intermedium (Seem.) Triana et Planch.

Árbol o arbusto 3-6 m. Fl amarilla. Oct. SBC. ANZ 816; ASM $55,682$.

\section{HERNANDIACEAE}

Gyrocarpus mocinnoi Espejo. Árbol 3-12 m. FI amarilla o verde. Oct.-dic. SBC, SMS. GJ 1775, 1846; ANZ 1293, 1306; SS 5613.

\section{HIPPOCRATEACEAE}

Hippocratea celastroides Kunth. Bejuco. FI verde. Feb.-abr. SBC. G) 1829, 2025; SAY 5448, 5533.

Hippocratea volubilis L. Bejuco. FI verde. May. SBC. ASM 828.

Hippocratea mexicana Miers. Bejuco o arbusto escandente. FI blanca verdosa. Feb.-mar. Sa, SBC. ASM 1272, 705; SAY 5396.

\section{HYDROPHYLLACEAE}

Wigandia urens (Ruiz et Pav.) Kunth. Arbusto 1-4 m. Fl lila o morada. May.-oct. SBC, SBC2, Ga. ANZ 538, 708; ASM 261, 624,671 .

\section{JULIANIACEAE}

Amphipterygium adstringens (Schltdl.) Standl.

Árbol o arbusto 2-12.5 m. Fl blanca o amarilla. Jun.-ago. SBC, Ma. GJ 1737; ANZ 476, 500; ASM 1060, 174.

\section{KRAMERIACEAE}

Krameria cuspidata C.Presl. Arbusto 40-80 cm. Flor rosa o morada. Todo el año. SBC, Sa. ANZ 1023, 1382; SS 5909; ASM 576, 94.

Krameria revoluta O.Berg. Arbusto rastrero. FI roja o rosa mexicano. Sept.feb. SBC, Sa. ANZ 833; ASM 1249, 510; SAY 4238.

\section{LAMIACEAE}

Hyptis mociniana Benth. Arbusto $60 \mathrm{~cm}-2.5 \mathrm{~m}$. Fl blanca o verde. Dic.-feb. SBC, Ga. ANZ 714, 872; ASM 608; SAY $5278,5340$.

Hyptis suaveolens (L.) Poit. Arbusto $50 \mathrm{~cm}-1.5 \mathrm{~m}$. Fl blanca, morada o azul. Sept.-nov. SBC. ANZ 1231, 1272; ASM 104, 195, 264.

Hyptis tomentosa Poit. Arbusto $60 \mathrm{~cm}-3 \mathrm{~m}$. Fl lila o morada. Ene.-may. SBC, SBC2. GJ 2169; ANZ 1021; ASM 586, 805, 827.

\section{LEGUMINOSAE}

Acacia cochliacantha Humb. et Bonpl. ex Willd. Árbol 2-8 m. Fl amarilla. Abr.-jul. SBC, SBC2. GJ 2182; ANZ 1381, 785; ASM 1402; SAY 4385.

Acacia collinsii Saff. Árbol o arbusto 2-6 m. Fl amarilla. Oct.jun. SBC, SBC2, Sa. AÁH 95; GJ 2209; ASM 1411, 196; SAY 4229 . 
Acacia cornigera (L.) Will. Arbusto $4 \mathrm{~m}$. Fl amarilla. Abr. SBC2. ANZ 1326.

Acacia hindsii Benth. Árbol o arbusto 3-8 m. FI amarilla. Feb.-mar. SBC, SMS. SS 5558; SAY 5308, 5315, 5333.

Acacia macracantha Humb. et Bonpl. ex Willd. Árbol o arbusto 3-8 m. FI amarilla. Jun.-nov. SBC. GJ 2370; ANZ 1479, 976; ASM 446, 655.

Acacia picachensis Brandegee. Árbol 3-7 m. Fl blanca. Mar.oct. SBC, SBC2. ASM 263.

Acacia sp. Árbol 6 m. Fr café. Dic. SBC. ANZ 775.

Acaciella angustissima (Mill.) Britton et Rose Árbol o arbusto 80 cm-11 m. Fl blanca. May.-oct. SBC, SBC2. GJ 1845; ANZ 1082; ASM 1038, 1067, 330.

Acaciella villosa (Sw.) Britton et Rose. Arbusto $80 \mathrm{~cm}-2 \mathrm{~m}$. FI blanca. Feb. SBC. SAY 5411.

Adenopodia oaxacana M.Sousa. Bejuco o arbusto escandente. FI amarilla. Oct.-ene. SBC. ANZ 546, 710; ASM 1195; SAY 5337, 5358.

Aeschynomene americana L. Hierba $60-70 \mathrm{~cm}$. Fl lila. Oct. SBC. SS 5445; ASM 212.

Aeschynomene compacta Rose. Árbol o arbusto $60 \mathrm{~cm}-3 \mathrm{~m}$. FI amarilla. Oct. SBC. GJ 1793, 1802; ANZ 855; SS 5566; ASM 571.

Aeschynomene fascicularis Schltdl. et Cham. Hierba o arbusto $80 \mathrm{~cm}-3 \mathrm{~m}$. Fl amarilla. Jun.-oct. SBC. ASM 273, 294.

Albizia adinocephala (Donn.Sm.) Britton et Rose. Árbol 7-12 m. Fl amarilla. Nov. SBC. ANZ 555, 560; ASM 1277.

Albizia occidentalis Brandegee. Árbol 4-12 m. Fl blanca. Mar.may. SBC, SBC2. ANZ 1006, 1017; SS 5426; ASM 1003, 1029.

Andira inermis (W.Wright) Kunth ex DC. Árbol 5-18 m. Fl lila o morada. Feb.-abr. SBC. ASM 1311, 1368; SAY 5522; KV 744.

Apoplanesia paniculata C.Presl. Árbol 4-8.3 m. Fl blanca. Oct.-nov. SBC. GJ 1741; ANZ 534, 771; SS 5385; ASM 189.

Bauhinia divaricata L. Árbol o arbusto 1.8-5 m. Fl blanca. Oct.-may. SBC, SBC2. ANZ 1322, 552; ASM 658, 674; SAY 4407.

Brongniartia bracteolata Micheli. Árbol o arbusto 1-4 m. FI vino. Jun. SBC. SAY 5610, 5636.

Caesalpinia eriostachys Benth. Árbol o arbusto 2.5-6 m. FI amarilla. Jun.-ago. SBC. ME 6463.

Caesalpinia hughesii G.P.Lewis. Árbol 5-8 m. FI amarilla. Feb.-abr. SBC, Ma. ANZ 1344, 967; ASM 1406, 647.
Caesalpinia mollis (Kunth) Spreng. Arbusto $1 \mathrm{~m}$. Fr café. Oct. SBC. ANZ 482.

‡ Caesalpinia pulcherrima (L.) Sw. Árbol o arbusto 2-6 m. FI roja o anaranjada. Ago.-ene. SBC, Ga. AÁH 94; ANZ 1195, 1283; ASM 1028, 396.

Caesalpinia sclerocarpa Standl. Árbol 8-16 m. Fl amarilla. Jun.-sept. SBC. ANZ 1080, 1196; SS 5466; ASM 1031.

Caesalpinia velutina (Britton et Rose) Standl. Árbol 3-8 m. Fl amarilla. Abr.-oct. SBC, Sa, VD. G) 1817, 1895; ANZ 1216; ASM 1452, 176.

Caesalpinia sp. Árbol 3-8 m. Fr verde o negro. Feb. SBC. SAY 5330.

Calliandra calothyrsus Meisn. Arbusto 2.5-5 m. FI roja. Sept. SBC. ASM 593; SAY 4275.

Calliandra houstoniana (Mill.) Standl. Árbol $5 \mathrm{~m}$. Botones. Oct. SBC. ASM 293.

Calliandra tergemina (L.) Benth. Arbusto 1-3 m. Fl amarilla o blanca. Mar.-jul. SBC. SS 5266; SAY 5665.

Calopogonium mucunoides Desv. Bejuco. Fr verde. Feb. SBC. SS 5551.

Canavalia acuminata Rose. Bejuco. Fl lila o azul. Nov.-feb. SBC. ANZ 666; ASM 465; SAY 5440.

Canavalia brasiliensis Mart. ex Benth. Bejuco. FI rosa o morada. Ago.-abr. SBC, SBC2, Ga. ANZ 1244; ASM 345, 613; SAY 4207, 5496.

Canavalia sp. Bejuco. Fl café y amarillo. Nov. SBC. ASM 329.

Centrosema molle Mart. ex Benth. Bejuco. Fr verde y café. Ene.-feb. SBC. ANZ 948; SAY 5275.

Centrosema plumieri (Turpin ex Pers.) Benth. Bejuco. Fl blanca con morado. Dic.-abr. SBC, SBC2. ANZ 694, 865; ASM 1427; SAY 5322.

Centrosema sp. Bejuco. Fr verde. Feb. SBC. SS 5549.

Chaetocalyx scandens (L.) Urb. Bejuco. FI amarilla. Sept. SBC. ASM 1094, 533.

Chamaecrista fagonioides (Vogel) H.S.Irwin et Barneby var. fagonioides. Hierba rastrera $60 \mathrm{~cm}$. Fl amarilla o anaranjada. Feb.-abr. SBC, Sa. ANZ 1379; ASM 1271.

Chamaecrista flexuosa (L.) Greene var. no determinada. Hierba 40-60 cm. Fl café, amarilla o salmón. Abr.-jun. SBC. ASM 88.

Chamaecrista flexuosa (L.) Greene var. texana (Buckley) H.S.Irwin et Barneby. Hierba $40 \mathrm{~cm}$. Fl amarilla o salmón Feb.-may. SBC. ASM 1250; SS 5942. 
Chamaecrista hispidula (Vahl) H.S.Irwin et Barneby. Hierba rastrera. FI amarilla a anaranjada. Sept.-oct. Sa. SAY 4241; ASM 92.

Chamaecrista nictitans (L.) Moench. var. no determinada. Hierba sufrutescente 30-60 cm. FI amarilla. Sept.-nov. SBC, SBC2. SAY V. 5021, 5173; ANZ 559; ASM 66, 363.

Chamaecrista nictitans (L.) Moench var. jaliscensis (Greenm.) H.S.Irwin et Barneby. Hierba 25 cm-1 m. Fl. amarilla. Sept.dic. SBC, SBC2. ANZ 686, 1266; ME 7274; ASM 464, 1202.

Chamaecrista rotundifolia (Pers.) Greene var. rotundifolia. Hierba 50 cm. Fl. amarilla. Oct. SBC. ASM 1133.

Chloroleucon mangense (Jacq.) Britton et Rose

Árbol 4-12 m. Fl. amarilla o blanca. May.-ene. SBC, SBC2. G) 1776, 2183; SS 5387, 5939; SAY 5467.

Coursetia caribaea (Jacq.) Lavin. Arbusto $20 \mathrm{~cm}-1.5 \mathrm{~m}$. Fl. blanca, crema o amarilla. Sept.-may. SMS, SBC, SBC2. GJ 2222, 2246; ASM 248, 360; SAY 5063.

Coursetia glandulosa A.Gray. Árbol 6 m. Fl. blanca con líneas moradas. Dic. SBC. GJ 1909.

Crotalaria cajanifolia Kunth. Hierba sufrutescente $1 \mathrm{~m}$. Fl. amarilla. Ago.-sept. SBC, Ga. ASM 1008; SAY 4426.

Crotalaria longirostrata Hook. et Arn. Hierba sufrutescente 1 m. Fl amarilla. Oct. SBC. SAY 5205.

Crotalaria pumila Ortega. Hierba $60-80 \mathrm{~cm}$. Fl amarilla. Oct.nov. SBC. ASM 388; SS 5432.

Crotalaria sp. Árbol 3 m. Fl. amarilla. Ene. SBC. ANZ 947.

Dalbergia congestiflora Pittier. Árbol 5-7 m. Fl. amarilla. Ene. SBC. KV 712; ANZ 841.

Dalbergia glabra (Mill.) Standl. Árbol 2.5 m. Fl. blanca. Mar. SBC. ASM 729.

Dalbergia granadillo Pittier. Árbol 4-13 m. Fl. blanca-crema. May.-dic. SBC, SBC2. GJ 1947, 2184, 2195; SS 5938; ASM 46.

Desmanthus virgatus (L.) Willd. Hierba sufrutescente $1.5 \mathrm{~m}$. FI. blanca. Oct. SBC. SAY 5032.

Desmanthus sp. Hierba 60 cm. Fr. café seco. Abr. Ma. ANZ 1348.

Desmodium distortum (Aubl.) J.F.Macbr. Hierba sufrutescente $2 \mathrm{~m}$. Fr. verde y café. Ene. SBC. ANZ 945.

Desmodium glabrum (Mill.) DC. Hierba 70 cm.-1.5 m. Fl. amarilla, lila o café. Oct.-dic. SBC, SBC2. ASM 21, 351; ANZ 668.

Desmodium hookerianum D.Dietr. Hierba 1 m. Fl. lilas. Oct. SBC. SS 5406.
Desmodium infractum DC. Bejuco. Fl. morada. Oct. SBC. SS 5412.

Desmodium procumbens (Mill.) Hitchc. Hierba rastrera de 1 m. FI. blanca. Oct. SBC. ASM 1176, 1206.

Desmodium scorpiurus (Sw.) Desv. Hierba $20 \mathrm{~cm}$. o bejuco. Fl. verde. Ago.-sept. SBC, SBC2. ANZ 1204; ME 7037.

Diphysa americana (Mill.) M.Sousa. Arbusto o árbol 3.5-5 m. Fr. globoso café. Oct.-ene. SBC. GJ 1798; ASM 670.

Diphysa suberosa S.Watson. Arbusto 1.5-3 m. Fl. amarilla. Sept.-oct. SBC, Sa. ASM 95; SAY 4228.

Entadopsis polystachia (L.) Britton. Bejuco. Fr. verde. Dic.feb. SBC. ANZ 730, 937; SAY 5300.

Enterolobium cyclocarpum (Jacq.) Griseb. Árbol 7-18 m. Fl. blanca. Ene.-jun. SBC, SBC2. ASM 710, 715, 1407; KV 714; SAY 5512.

Eriosema grandiflorum (Schltdl. et Cham.) G.Don. Arbusto 1 m. Fl amarilla, fr verde. Feb. Sa. ASM 1269.

Erythrina lanata Rose. Árbol o arbusto 3-12 m. Fl. rosa claro. Jun.-feb. SBC, SBC2. SS 5570; ANZ 778, 1114; SAY 5154, 5604.

Galactia sp. Hierba rastrera. FI. morada. Nov. SBC. ANZ 520.

Gliricidia sepium (Jacq.) Kunth ex Walp. Árbol o arbusto 1-8 m. FI. rosa con amarillo y blanco. Oct.-abr. SBCE, VD, SBC, SBC2. SS 3797, 5626; SAY 5307, 5347.

Havardia campylacantha (L.Rico et M.Sousa) Barneby et J.W.Grimes. Árbol 5-7 m. Fr. verde. Ago.-sept. SBC, SBC2. ASM 1011; ANZ 1172; SAY 4299.

Heteroflorum sclerocarpum M.Sousa. Árbol 11 m. Fr. en vaina. Nov. SBC. GJ 1841.

Indigofera hirsuta L. Arbusto $50 \mathrm{~cm}-2 \mathrm{~m}$. Fl. rosa o roja. Ago.mar. SBC, SBC2. ANZ 523, 1174, 1267; SAY 5210; ASM 205.

Indigofera jamaicensis Spreng. Hierba sufrutescente $50 \mathrm{~cm}$. FI. naranja o roja. Jul.-dic. SBC. ASM 1005; ANZ 693; SAY 5745.

Indigofera lancifolia Rydb. Hierba sufrutescente $80 \mathrm{~cm}-3 \mathrm{~m}$. Fl. roja, rosa o durazno. Ago.-mar. SBC, SBC2, SMS. ASM 368, 402; ANZ 1134, 1247; SAY 4182.

Indigofera miniata Ortega. Hierba rastrera. Fl. salmón. Oct. SBC. SAY 5065.

Indigofera platycarpa Rose. Árbol o arbusto 3-4 m. Fl. guinda o roja. Sept.-oct. SBC, Sa. SAY 4242, 4287; ASM 100.

Leucaena lanceolata S.Watson var. lanceolata. Árbol 5 m. Fr. café. Feb. SBC. SAY 5437. 
Leucaena lanceolata S.Watson var. sousae (S.Zárate) C.E.Hughes. Árbol 5 m. Fl. blanca en cabezuela. Oct. SBC. ASM 191.

Leucaena leucocephala (Lam.) de Wit. Árbol 3-7 m. FI. blanca. Jul.-abr. SBC, SBC2. ASM 657, 1378; ANZ 564, 1240; SAY 5680.

Leucaena macrophylla Benth. subsp. istmensis C.E.Hughes. Arbusto 2.5 m. Fl. blanca. Ene. SBC. KV 705.

Lonchocarpus constrictus Pittier. Árbol o arbusto 3-8 m. Fl. morada o lila. Feb.-ago. SBC. KV 740; ME 6456, 7050; GJ 2388, 2632.

Lonchocarpus emarginatus Pittier. Árbol 3-8 m. Estéril. SBC. G) 1852, 1854.

Lonchocarpus eriocarinalis Micheli. Árbol 3-6 m. Fr. verde. Ene.-may. SBC, SBC2. ANZ 850; ASM 821; SAY 5699.

Lonchocarpus guatemalensis Benth. Árbol o arbusto 1-15 m. Fl. blanca con rosa o morada. Ene.-may. SBC, SBC2, SMS. SAY 5310, 5430; ANZ 980, 1016; GJ 2244.

Lonchocarpus hermannii M.Sousa. Árbol o arbusto 3-12 m. Fl. morada o rosa mexicano. Abr.-may. SBC, SBC2. ANZ 1001; GJ 2236; ASM 802.

Lonchocarpus lanceolatus Benth. Árbol o arbusto 4-20 m. Fl. guindas o moradas. Jul.-oct. SBC. GJ 1783, 1790; SAY 5689; ASM 518, 1143.

Lonchocarpus sericeus (Poir.) Kunth ex DC. subsp. palmeri (Rose) M.Sousa. Árbol o arbusto 3-7 m. Fl. morada. Oct.-feb. SBC. ANZ 433, 494, 796; SS 5576.

Lysiloma acapulcense (Kunth) Benth. Árbol 5 m. Fl. blanca. May. SBC. ASM 1486.

Lysiloma microphyllum Benth. Árbol 5-15 m. Fl. blanca. Jun.ago. SBC. GJ 1867, 1786; ASM 1621; ANZ 1290, 1314.

Macroptilium atropurpureum (Sessé et Moc. ex DC.) Urb. Bejuco. Fl. guinda, morada obscura o negra. Ene.-abr. SBC. ANZ 879, 911; ASM 1393.

Macroptilium longepedunculatum (Mart. ex Benth.) Urb. Hierba rastrera o bejuco. Fl. morada, púrpura intenso o roja obscura. Oct.-nov. SBC. ASM 198, 1173, 1200; ANZ 519, 692.

Mimosa acantholoba (Humb. et Bonpl. ex Willd.) Poir. var. no determinada. Arbusto. Fl. verde, blanca, guinda, amarilla o roja. Ago.-feb. SMS, SBC, SBC2, Sa. SAY 4183, 4243; AÁH 70; ANZ 1087, 1273.

Mimosa acantholoba (Humb. et Bonpl. ex Willd.) Poir. var. eurycarpa (B.L.Rob.) Barneby. Arbusto $2 \mathrm{~m}$. Fr. verde, rojo o café. Oct.-ene. SBC. ANZ 542, 834; ASM 211, 407, 662.
Mimosa albida Humb. et Bonpl. ex Willd. var. pochutlensis R.Grether. Arbusto 1.5 m. Fl. rosa. Ago. SBC. ASM 1010.

Mimosa antioquensis Killip ex Rudd var. isthmensis R.Grether. Árbol o arbusto 2-5 m. Fl. morada, rosa o blanca. Ago.-ene. SBC, SBC2, SMS, Sa. ANZ 705, 812; SAY 4176, 4233; ASM 35.

Mimosa arenosa (Willd.) Poir. Árbol 5 m. Fr. café. Nov. SBC. ANZ 529.

Mimosa pigra L. var. pigra. Arbusto 2 m. Fl. rosa. Ago. SBC. AÁH 54.

Mimosa pudica L. Hierba $30 \mathrm{~cm}$. Fl. de estambres rosas. Oct. SBC. ASM 64.

Mimosa robusta R.Grether. Arbusto escandente o arbusto 2 m. Fl. rosa. Oct.-nov. SBC. SAY 5086, 5299, 5327; ASM 408; ANZ 860.

Myrospermum frutescens Jacq. Árbol $8 \mathrm{~m}$. Fl blanca. Dic.ene. SBC. GJ 1758, 1759.

Nissolia chiapensis Rudd. Bejuco. FI. amarilla. Oct. SBC. ASM 52, 1185.

Nissolia fruticosa Jacq. var. fruticosa. Bejuco. Fl. amarilla. Oct. SBC. SAY 4343.

Phaseolus lunatus L. Bejuco. Fl. amarilla o morada. Ene.-abr. SBC. SS 5550; ASM 660, 1371, 1377B; ANZ 916.

Phaseolus microcarpus Mart. Hierba rastrera o bejuco. FI. morada, rosa, blanca, amarilla. Sept.-dic. SBC. ASM 85, 126; SAY 5147, 5238; SS 5451.

Piptadenia flava (Spreng. ex DC.) Benth. Bejuco, arbusto escandente o arbusto 2-4 m. Fl. amarilla. Oct.-nov. SBC. ASM 214, 285, 314; ME 6465; ANZ 524.

Piptadenia obliqua (Pers.) J.F. Macbr. Árbol o arbusto 3-12 m. Fl. café. Feb. SBC. GJ 1809, 1853; SAY 5354; ASM 516; ANZ 743.

Piscidia carthagenensis Jacq. Árbol o arbusto 1.5-14 m. Fl. blancas o rosa con centro verde. Feb.-abr. SBC, Ma. GJ 1756, 1870; SAY 5311, 5441; ASM 1275.

Pithecellobium dulce (Roxb.) Benth. Árbol o arbusto 4-7 m. FI. café o blanca. Dic.-abr. SBC. ANZ 745; ASM 521, 1310, 1401.

Pithecellobium lanceolatum (Willd.) Benth. Árbol 2-6 m o arbusto escandente. Fl. blanca, verde o rosa mexicano. Oct.may. SBC. ASM 257, 258; ANZ 441, 1354; KV 730.

Pithecellobium seleri Harms. Árbol o arbusto 2-6 m. Fl. blanca. Oct.-abr. SBC. SS 5441; ASM 348, 1382. 
Pithecellobium sp. Árbol 3 m. Fr. seco. Jun. SBC. SAY 5531.

Poeppigia procera C.Presl. Árbol 5-15 m. FI. amarilla. Jun.sept. SBC, SBC2. SAY 4309, 4408; ANZ 1523; ME 7348.

Prosopis juliflora (Sw.) DC. Árbol o arbusto 2-6 m. Fl. blanca o amarilla. Nov.-abr. SBC, SBCE, SBce2, VD. SAY 4388, 4450; ANZ 526, 990; ASM 1321.

Pterocarpus acapulcensis Rose. Árbol 4-12 m. Fl. amarilla. Oct.mar. SBC. SS 5438; SAY 5324; ASM 714; GJ 2382; ANZ 1386.

Pterocarpus rohrii Vahl. Árbol 3-10 m. Fl. amarilla. May. SBC. KV 742; SAY 5525.

Ramirezella strobilophora (B.L.Rob.) Rose. Bejuco. Fr. verde. Feb. SBC. ANZ 981.

Rhynchosia minima (L.) DC. Hierba rastrera o bejuco. Fl. amarilla o morada. Oct.-mar. SBC, Pz. SS 5403; ASM 466, 469; ANZ 557; SAY 5349.

Rhynchosia precatoria (Humb. et Bonpl. ex Willd.) DC. Bejuco. Fr. café con semilla roja con negro. Dic.-jun. SBC. ASM 1447, 1605; ANZ 813, 1009.

Rhynchosia sp. Bejuco. Fl. morada. Oct. SBC. ASM 1191.

Senna atomaria (L.) H.S.Irwin et Barneby. Árbol o arbusto 80 cm-8 m. Fl. amarilla o anaranjada. Todo el año. SBC, SBC2, SBCE, Sa. ANZ 982, 996; SS 5891; ASM 40, 628.

Senna cobanensis (Britton) H.S.Irwin et Barneby. Arbusto $30 \mathrm{~cm}$ 1.5 m. Fl. amarilla. Ene. SBC. ANZ 880, 894, 942; SAY 5295.

Senna fruticosa (Mill.) H.S.Irwin et Barneby. Arbusto escandente, arbusto o árbol 1-4 m. Fl. amarilla. Ago.-oct. SBC. ANZ 941, 1175; ASM 419, 949; ME 7045.

Senna holwayana (Rose) H.S.Irwin et Barneby. var. holwayana. Arbusto 50 cm-1 m. Fl. amarilla. Dic.-feb. SBC. SS 5515, 5562.

Senna mollissima (Humb. et Bonpl. ex Willd.) H.S.Irwin et Barneby var. no determinada. Árbol o arbusto 3-8 m. Fl. amarilla. Jul.-oct. SBC. GJ 1781; ASM 72; SAY 5677.

Senna mollissima (Humb. et Bonpl. ex Willd.) H.S.Irwin et Barneby var. glabrata (Benth.) H.S.Irwin et Barneby. Arbusto 2-3 m. Fl. amarilla. Abr.-jun. SBC, SBC2. SAY 5507, 5529; ASM 1300, 1370; ANZ 1359.

Senna nicaraguensis (Benth.) H.S.Irwin et Barneby. Árbol 3 m. Fl. amarilla. Ene. SBC. ANZ 955.

Senna obtusifolia (L.) H.S.Irwin et Barneby. Hierba 50 cm. Fl. amarilla. Feb. SBC. ASM 1319.

Senna occidentalis (L.) Link. Arbusto escandente o arbusto 1 m. FI. amarilla. Oct.-dic. SBC, SBC2. ANZ 696, 1302; ASM 1112, 1137.
Senna pallida (J.Vahl) H.S.Irwin et Barneby. var. pallida. Arbusto $50 \mathrm{~cm}-2 \mathrm{~m}$. Fl. amarilla. Nov.-mar. SBC, SBC2. SAY 5450; ASM 420, 482, 676; ME 6455.

Senna pallida (J.Vahl) H.S.Irwin et Barneby var. geminiflora H.S.Irwin et Barneby. Hierba $40 \mathrm{~cm}$. Fl. amarilla. Feb. SBC. ASM 1303.

Senna reticulata (Willd.) H.S.Irwin et Barneby. Árbol o arbusto 1-3 m. Fl. amarilla o anaranjada. Dic.-ene. SBC, Ga. KV 657; SAY 4428; ANZ 712, 857; ASM 604.

Senna skinneri (Benth.) H.S.Irwin et Barneby. Arbusto 2 m. Fl. amarilla. May. SBC. ASM 759.

Senna uniflora (Mill.) H.S.Irwin et Barneby. Hierba rastrera o hierba 30-70 cm. Fl. amarilla. Ago.-nov. SBC, SBC2. ASM 1107, 1147; AÁH 77; ME 7285; ANZ 556.

Senna sp. Árbol 6 m. FI. amarilla. Sept. SBC. SAY 4262.

Sesbania herbacea (Mill.) McVaugh. Hierba robusta $3 \mathrm{~m}$. Fl. amarilla. Oct. SBC2. ANZ 1207.

Stizolobium pruriens (L.) Medik. var. utilis Wall. ex Wight. Bejuco. Fr. seco. Abr. SBC. ASM 1414.

Stylosanthes humilis Kunth. Hierba $40 \mathrm{~cm}$. Fl. anaranjada. Oct. SBC. SAY 5082.

Stylosanthes viscosa (L.) Sw. Hierba rastrera. Fl. amarilla. Oct. SBC. ANZ 480.

Tephrosia macrantha A.Rob. et Greenm. ex Pringle. Arbusto $1.5 \mathrm{~m}$. Fl. blanca y rojiza. Oct. SBC. SAY 5211.

Tephrosia vicioides Schltdl. Hierba rastrera. Fl. rosa mexicano. Nov. SBC. ASM 511.

Tephrosia sp. Arbusto. Fl. blanca con un pétalo moteado de café. Oct. SBC. ANZ 491.

Teramnus uncinatus (L.) Sw. Bejuco. Fr. de parte media amarilla y borde café. Dic.-ene. SBC. ANZ 732, 871.

Vigna adenantha (G.Mey.) Maréchal, Mascherpa et Stainer. Bejuco. FI. lila. Abr. SBC. ASM 1443.

Vigna speciosa (Kunth) Verdc. Bejuco. Fl. morada. Dic. SBC. ANZ 695.

Vigna umbellata (Thunb.) Ohwi et Ohashi. Bejuco. FI. amariIla. Oct. SBC. SAY 5085.

Zapoteca formosa (Kunth) H.M.Hern. subsp. formosa. Árbol o arbusto 3-5 m. Fr. verde. Nov.-dic. SBC. SS 5526; ASM 375.

Zapoteca formosa (Kunth) H.M.Hern. subsp. rosei (Wiggins) H.M.Hern. Arbusto 1-1.5 m. Fl. roja. Sept. SBC, SBC2, Ga. ASM 614, 1076; SS 5439; ANZ 1303. 
Zapoteca media (M.Martens et Galeotti) H.M.Hern. Hierba rastrera. Fr verde. Oct. SBC. ANZ 472.

\section{LOASACEAE}

Gronovia scandens L. Bejuco. Fl. verde amarillenta. Jul.nov. SMS, SBC, SBC2. SAY 4174, 4180; AÁH 68; ASM 410, 1111.

Mentzelia aspera L. Hierba 30-80 cm. Fl. amarilla o anaranjada. Sept.-nov. SBC, SBC2. ASM 25, 278; SAY 4399, 5028; ANZ 687.

\section{LYTHRACEAE}

Adenaria floribunda Kunth. Arbusto escandente o arbusto 23 m. Fl. blanca. Jun.-jul. SBC, SBC2. ME, 6973; ANZ 1487; SAY 5176.

Ammania coccinea Rottb. Hierba 60 cm. Fr. rojo. Abr. Ga. ANZ 1373.

\section{MALPIGHIACEAE}

Bunchosia caroli W.R.Anderson. Árbol 8 m. Fr. verde. Oct. SBC. ASM 240.

Bunchosia discolor Turcz. Árbol o arbusto 3-8 m. Fr. amariIlo-naranja. Oct.-dic. SBC. GJ 1782, 1834, 1924; ANZ 1297.

Bunchosia palmeri S.Watson. Arbusto escandente, arbusto o árbol 1.5-7 m. Fl. amarilla oct.-nov. SBC, Ga. ANZ 502, 577, 986; ASM 322, 600.

Byrsonima crassifolia (L.) Kunth. Árbol 3-5 m. Fl. amarilla, naranja o roja. Abr.-oct. SBC, SBC2, Sa. ANZ 1018, 1512; SAY 4237, 5679; GJ 2178.

Galphimia arenicola C.E.Anderson. Arbusto escandente o arbusto 1 m. Fl. amarilla. Ago.-nov. SBC. ASM 965.

Galphimia tuberculata (Rose) Nied. Arbusto $1 \mathrm{~m}$. Fl. amarilla. Sept. SBCE. SAY 4453.

Heteropterys laurifolia (L.) A.Juss. Árbol o arbusto 2-16 m. Fl. amarilla o café. Feb.-jun. SBC, SBC2, Ma. ASM 1317, 1450; SAY 5497.

Heteropterys palmeri Rose. Arbusto 4-5 m. Fl. rosa o pétalos blancos con base rosa y glándulas del cáliz rojas. Ene.-feb. SBC, Sa. ASM 589, 1270; ANZ 838, 843.

Lasiocarpus sp. Árbol 8 m. Fl. secas. May. SBC. GJ 2218.

Malpighia glabra L. Arbusto 2 m. Estéril. SBC. GJ 1935.

Malpighia mexicana A.Juss. Arbusto $3.5 \mathrm{~m}$. Fl. lila. Jul. SBC. SAY 5696, 5726.

Malpighia ovata Rose. Arbusto decumbente o arbusto $50 \mathrm{~cm}$ 2 m. Fl. rosa. Mar.-oct. SBC, SBCE. ASM 120, 148; SS 5575, 5621; ANZ 473.
Malpighia sp. Arbusto $1.5 \mathrm{~m}$. Fl. morada. Jun. SBC. SAY 5545.

Stigmaphyllon lindenianum A.Juss. Bejuco. Fl. amarilla. Mar.abr. SBC. ANZ 793, 992; ASM 711, 1405; KV 749.

Tetrapterys heterophylla (Griseb.) W.R.Anderson. Árbol 4 m. FI. amarilla. Ene. SBC. ANZ 952.

Tetrapterys mexicana Hook. et Arn. Bejuco. FI. amarilla. Feb. SBC. SAY 5309.

\section{MALVACEAE}

Abutilon bracteosum Fryxell. Hierba rastrera, hierba o sufrútice $60 \mathrm{~cm}-2 \mathrm{~m}$. Fl. amarilla. Oct.-ene. SBC. ASM 229, 287, 333, 1194; ANZ 587.

Anoda cristata (L.) Schltdl. Bejuco. Fl. amarilla. Nov. SBC. ASM 378.

Anoda thurberi A.Gray. Hierba $20 \mathrm{~cm}$. FI. lila. Oct. SBC. SAY 5144.

Bastardiastrum gracile (Hochr.) D.M.Bates. Arbusto 1.5 m. Fl. amarilla. Dic. SBC. ANZ 711.

Gossypium aridum (Rose et Standl.) Skovst. Árbol $5 \mathrm{~m}$. Fl. rosa. Feb. SBC. SS 5557.

Herissantia crispa (L.) Brizicky. Hierba rastrera o bejuco. Fl. amarilla, blanca o crema. Sept.-ene. SBC, SBC2. ANZ 729, 765; SS 5463, 5536; SAY 5194.

Hibiscus phoeniceus Jacq. Arbusto $40 \mathrm{~cm}-4 \mathrm{~m}$. FI. roja o anaranjada. Oct.-abr. SBC. ASM 216, 1308, 1433, 1448; ME 6451.

Kosteletzkya depressa (L.) O.J.Blanch., Frixell et D.M.Bates. Hierba 50 cm-1 m. Fl. rosa. Mar. Pz. SS 5619; ASM 1335.

Malachra capitata (L.) L. Hierba $40 \mathrm{~cm}-1 \mathrm{~m}$. Fl. amarilla. Abr.-sept. SBC, Ga. ANZ 1339; ME 6975; SAY 4419.

Malachra fasciata Jacq. Arbusto $40 \mathrm{~cm}-2 \mathrm{~m}$. Fl. rosa nov. SBC. ANZ 568, 1336, 1353.

Malvastrum americanum (L.) Torr. Hierba $50 \mathrm{~cm}-2 \mathrm{~m}$. Fl. anaranjanda o amarilla. Oct.-dic. SBC. ANZ 704, 1334; ASM 220, 1423 .

Malvastrum coromandelianum (L.) Garcke. Hierba $60 \mathrm{~cm}$. Fl. amarilla. Abr. SBC. ASM 1421.

Malvaviscus arboreus Cav. Arbusto 80 cm-2 m. Fl. roja. May.oct. SBC. SS 5448, 5537; KV 721.

Sida acuta Burm.f. Hierba 40-80 cm. Fl. amarilla, anaranjada, salmón o blanca. Jul.-oct. SBC, SBC2, Ga. SAY 4284, 5040; ASM 62, 84; ANZ 1220. 
Sida aggregata C.Presl. Arbusto $1 \mathrm{~m}$. Fl. naranja. Oct. SBC. SAY 5175; ASM 519.

Sida ciliaris L. Hierba rastrera o hierba de 20-0 cm. Fl. café, salmón o coral. Sept.-oct. SBC, SBC2. ANZ 1203; ASM 37; SAY 4314, 5071, 5623b.

Sida glabra Mill. Arbusto 1.5 m. Fl. anaranjada. Oct. SBC. ANZ 437.

Sida rhombifolia L. Arbusto $30 \mathrm{~cm}-1 \mathrm{~m}$. Fl. naranja, amarilla o coral. Oct.-ene. SBC, Ga. ANZ 566, 854; SAY 4436; ASM 208, 392.

\section{MELIACEAE}

Cedrela salvadorensis Standl. Árbol 10 m. Fl. verdosa. Feb. SBC. SS 5573.

₹ Melia azedarach L. Árbol $3 \mathrm{~m}$. Fr. verde. Jun. SBC2. ANZ 1524.

Swietenia humilis Zucc. Árbol 6-12 m. Fl. amarilla. Abr. SBC, SBC2. SS 5380; ANZ 1007, 1159; ASM 59, 556.

\section{MENISPERMACEAE}

Hyperbaena mexicana Miers. Árbol, arbusto o arbusto escandente 2-3 m. Fl. amarilla. Mar. SBC. ANZ 497, 446; ASM 683, 713; SAY 4413.

\section{MOLLUGINACEAE}

Mollugo verticillata L. Hierba $10-60 \mathrm{~cm}$. Fl. blanca o verde. Jun.sept. SBC, SBC2. ASM 939, 1034; ANZ 1234, 1482; SAY 4317.

\section{MORACEAE}

Dorstenia drakena L. Hierba $20 \mathrm{~cm}$. Fl. verde. Jun.-ago. SBC2. ANZ 1527; ASM 909; SAY 5588, 5634.

Ficus cotinifolia Kunth. Árbol 8-25 m. Fr. verde. Jun.-feb. SBC, SBC2. SAY 5190, 5361; GJ 2374; ANZ 1164, 1516.

Ficus insipida Willd. Árbol 5-15 m. Fr. verde. Sept.-ene. Ga, SBC. SAY 4424; KV 695.

Ficus trigonata L. Árbol 10-20 m. Sícono verde con puntos claros. SBC. ASM 1156; ME 7562; SS 5614.

Maclura tinctoria (L.) D.Don ex Steud. Árbol o arbusto 4-14 m. Fr. verde. Ago. SBC, SBC2. G) 1849; ASM 954; ANZ 1069, 1120.

Trophis racemosa (L.) Urb. Árbol 4-6 m. Fl. verde. Feb. SBC. ASM 1225, 1237.

\section{NYCTAGINACEAE}

Boerhavia coccinea Mill. Hierba 30-70 cm. Fl. guinda, roja o café. Abr.-oct. SBC, SBC2. SAY 5035; ASM 57, 199, 787; ANZ 713.

Boerhavia diffusa L. Hierba 40-50 cm. Fl. morada. Abr. SBC. ASM 1362, 1385.
Boerhavia erecta L. Hierba $30-80 \mathrm{~cm}$. FI. verde, blanca y guinda. Mar.-sept. SBC, SBC2, SBce2, SMS. ANZ 1233a, 1499; AÁH 79; SAY 4181, 4200.

Commicarpus scandens (L.) Standl. Hierba $80 \mathrm{~cm}$. Fr. verde. Dic. SBC. ANZ 808.

Mirabilis laevis (Benth.) Curran. Hierba $20-80 \mathrm{~cm} ., \mathrm{fl}$ rosa mexicano, lilas o rosas. Ago.-oct. SBC. ASM 114, 152; ANZ 1043, 1044.

Mirabilis sp. Arbusto 1 m. Fl. rosa-guinda. Oct. SBC. SAY 5051.

Neea psychotrioides Donn.Sm. Árbol 4-7 m. Botones blancos. Jul. SBC. GJ 1797, 1828 2369; SAY 5627.

Okenia hypogaea Schltdl. et Cham. Hierba rastrera. Fl. morada o rosa intenso. Ago.-oct. SBC, SBC2, SBCE. ASM 182; ANZ 1100; SAY 4445.

Pisonia aculeata L. Arbusto decumbente 1-3 m. Fl. morada, rojiza, rosa o café. Sept.-mar. SBC, SBC2, SBCE, SBce2. G) 1887, 1942; ASM 142, 179; SAY 4398.

Pisonia macranthocarpa (Donn.Sm.) Donn.Sm. Bejuco. Fl. amarillo-verdosa. Ene. SBC. SS 3809; ANZ 1188.

Salpianthus arenarius Humb. et Bonpl. Arbusto o arbusto escandente $40 \mathrm{~cm}-1.5 \mathrm{~m}$. Fl. verde, rosa, blanca o café. Oct.may. SBC, SBC2, SBCE, VD. ANZ 471, 508; ASM 449, 522; SAY 5397.

Salpianthus purpurascens (Cav. ex Lag.) Hook. et Arn. Arbusto decumbente 1-7 m. Fl. verde, amarilla o blanca. Feb.-abr. SBC, Ma, Pz, SMS, VD. ANZ 1332, 1338; ASM 1340, 697; SAY 5374.

Torrubia linearibracteata (Heimerl) Standl. Árbol o arbusto 34 m. Fl. morada. Oct. SBC. ASM 132; SAY 5536.

Torrubia macrocarpa Miranda. Árbol 2.5-5 m. Fl. verde. May. SBC, SBC2. GJ 2174, 2191; SS 5886, 5901; ASM 773.

OLACACEAE

Schoepfia schreberi J.F.Gmel. Árbol o arbusto 1.5-3 m. Fl. roja o café. Feb.-mar. VD, SBCE, SBC, Sa. SAY 5381; SS 5611, 5627; ASM 1264.

Ximenia americana L. Árbol o arbusto 1-5 m. Botones verdes. May. SBC, SBC2. SS 5898, 5919; ANZ 1024; GJ 2173.

\section{ONAGRACEAE}

Ludwigia erecta (L.) H.Hara. Hierba $60 \mathrm{~cm}-1 \mathrm{~m}$. Fl. amarilla. Ago.-sept. SMS, SBC. SAY 4190; ASM 995.

Ludwigia octovalvis (Jacq.) P.H.Raven. Arbusto 1 m. Cáliz verde y café. Ene. SBC. ANZ 888.

OXALIDACEAE

Oxalis yucatanensis (Rose) R.Knuth. Hierba sufrutescente 10- 
$40 \mathrm{~cm}$. Fl. amarilla. Abr.-oct. SBC, SBC2. SAY 5061, 5686; ASM 1119, 1420; ANZ 448.

\section{PASSIFLORACEAE}

Passiflora coriacea Juss. Bejuco. FI. verde. Nov.-abr. SBC. ANZ 735, 1025; SS 5507; ASM 503; SAY 5335.

Passiflora filipes Benth. Bejuco. FI. blanca o verde con amarillo. Oct. Ga. SAY 4438; ASM 225, 256.

Passiflora foetida L. var. no determinada. Bejuco, hierba o hierba rastrera. Fl. blanca o verde con centro morado. May.oct. SBC, SBC2. ASM 43, 757; SS 5493, 5911; SAY 5720.

Passiflora foetida L. var. gossypiifolia (Desv. ex Ham.) Mast. Bejuco. Fr. verde. Jul. SBC. ME 6921.

Passiflora mexicana Juss. Bejuco. FI. verde. Dic. SBC. ANZ 802, 1318; SS 5578.

\section{PEDALIACEAE}

Martynia annua L. Hierba robusta o hierba $40 \mathrm{~cm}-2 \mathrm{~m}$. Fl. blanca con manchas violetas. Jul.-oct. SBC, SBC2. AÁH 76; SAY 4282, 5693; ANZ 1083, 1102.

\section{PHYTOLACCACEAE}

Agdestis clematidea DC. Bejuco. Fl. blanca o verde. Jul.-oct. SBC, SBC2, SMS. SAY 4375, 5583; ANZ 496, 1065; ASM 262.

Petiveria alliacea L. Hierba 50-80 cm. Fl. blanca. Ago.-oct. SBC. ASM 255, 915; SAY 5201; ANZ 454, 1051.

Rivina humilis L. Hierba 30-80 cm. Fl. blanca. Abr.-oct. SBC, SBC2, SBCE. ASM 75, 246; ANZ 450, 1074; SAY 4384.

Trichostigma octandrum (L.) H.Walter. Bejuco. Fr. verde. Abr. SBC. ASM 1429, 1445.

\section{PLUMBAGINACEAE}

Plumbago scandens L. Hierba $50 \mathrm{~cm}$. Fl. blanca. Oct. SBC. SAY 5200.

\section{POLEMONIACEAE}

Loeselia ciliata L. Hierba. Fl. crema. Dic. SBC. ANZ 721.

Loeselia glandulosa (Cav.) G.Don. Hierba sufrutescente 75 cm. Fl. amarilla. Oct. SBC. SS 5437.

\section{POLYGALACEAE}

Securidaca sylvestris Schltdl. Bejuco. Fl. lila. Mar. SBC. ASM $730,1325$.

\section{POLYGONACEAE}

Antigonon cinerascens M.Martens et Galeotti. Bejuco. Fl. rosa o rosa mexicano. Sept.-nov. SBC. ANZ 434, 549; SAY 5263; ASM 370, 1089.

Antigonon flavescens S.Watson. Arbusto escandente o bejuco. Fl. blanca o amarilla pálido. May.-dic. SBC, SBC2, SBce2. SAY 4307, 5116; SS 5399, 5495; ANZ 492.
Coccoloba liebmannii Lindau. Árbol o arbusto 1-6 m. Fl. verde, blanca o amarilla. Jun.-dic. SBC, SBC2, Sa, SBCE. SAY 4263, 5241; GJ 1776-a, 1875; ASM 493.

Podopterus cordifolius Rose et Standl. Arbusto escandente o bejuco. FI. lila. Oct. SBC. SAY 5191, 5544; GJ 1866, 1883; ASM 127.

Podopterus mexicanus Bonpl. Árbol, arbusto o arbusto escandente 2-4 m. Fr. blanco con rojo, rosa azul o morado. Mar.-jun. SBC, SBC2, SBCE. GJ 1944a; ASM 1357; ANZ 1331; SAY 5502, 5539.

Polygonum segetum Kunth. Hierba $80 \mathrm{~cm}$. Fl. blanca con tonos rosas. Humedal. ANZ 1374.

Ruprechtia fusca Fernald. Árbol o arbusto 3-13 m. FI. verde o café. Nov. SBC, SBC2. ASM 423, 500; SAY 5332; ANZ 1300; G) 1777.

Ruprechtia pallida Standl. Árbol 5-7 m. Fr. verde. Oct. SBC. SS 5469; ASM 1942.

Ruprechtia standleyana Cocucci. Árbol 5 m. Fl. café. Ene. Ga. ASM 622.

PORTULACACEAE

Portulaca oleracea L. Hierba $60 \mathrm{~cm}$. Fl. blanca. Jul. SBC. SAY 5758.

Portulaca pilosa L. Hierba o hierba rastrera 10-60 cm. Fl. guinda, blanca, lila, morada, roja o rosa. Jun.-oct. SBC, SBC2. SAY 5070, 5704; AÁH 58; ASM 111, 1130.

Talinum paniculatum (Jacq.) Gaertn. Hierba 50-70 cm. Fl. blanca o lila. Jul.-ago. SBC. SAY 4311, 5192, 5595; ANZ 1046.

Talinum triangulare (Jacq.) Willd. Hierba $10-70 \mathrm{~cm}$. Fl. amarilla, lila, rosa o blanca con tonos rosas. May.-oct. SBC, SBC2. G) 2196, 2371; SAY 4306, 5031; ANZ 1047.

\section{RHAMNACEAE}

Colubrina elliptica (Sw.) Brizicky et W.L.Stern. Árbol 6 m. Fr. verde. Dic. SBC. ANZ 751.

Colubrina triflora Brongn. ex Sweet. Árbol 4-8 m. FI. amarillaverdosa. Oct. SBC, SMS. SS 5434; SAY 4221; GJ 1888, 1917.

Gouania eurycarpa Standl. Bejuco. Fl. amarillenta. Oct. SBC. SAY 5209, 5259, 5273; ASM 274, 513.

Gouania lupuloides (L.) Urb. Bejuco. Fl. amarilla. Nov. SBC. ASM 369; ANZ 1313; GJ 1754.

Karwinskia calderonii Standl. Árbol 7-8 m. Fl. amarilla. Ago. SBC. GJ 1763, 1800; ASM 951.

Karwinskia humboldtiana (Willd. ex Roem. et Schult.) Zucc. Árbol o arbusto 2.5-4 m. Fr. verde. Oct. SBC, SMS. SAY 5258; SS 5637. 
Ziziphus amole (Sessé et Moc.) M.C.Johnst. Árbol o arbusto 2-10 m. Fl. amarilla o verde. Ago. SBC, VD, SBCE. ASM 137, 288; SS 5471, 5530; GJ 2031.

\section{RHIZOPHORACEAE}

Rhizophora mangle L. Árbol $6 \mathrm{~m}$. Fl. blanca. Dic. SBC. SS 5543.

\section{RUBIACEAE}

Arachnothryx leucophylla (Kunth) Planch. Arbusto $50 \mathrm{~cm}-2$ m. FI. rosa. Ene.-feb. Ma2. ANZ 924, 892; SAY 5329.

Borreria laevis (Lam.) Griseb. Hierba 40-50 cm. Fl. blanca o translucida. Sept.-oct. SBC, SBC2. ANZ 447, 1214.

Borreria remota (Lam.) Bacigalupo et E.L.Cabral. Hierba 30 cm-1 m. Fl. blanca. Oct. SBC. SAY 5058; ASM 1121.

Borreria verticillata (L.) G.Mey. Hierba 30-60 cm. Fl. blanca. Sept. SBC, SBC2. ANZ 1237, 1257; ASM 536

Calycophyllum candidissimum (Vahl) DC. Árbol 6-18 m. FI blanca. Nov.-mar. SBC. ASM 371, 462; SAY 4341, 5217; SS 5520 .

Chiococca alba (L.) Hitchc. Arbusto, arbusto escandente o bejuco $80 \mathrm{~cm}-2 \mathrm{~m}$. Fl. amarilla o verde. Sept.-oct. SMS, SBC. SAY 4364, 5406; ANZ 465, 809; ASM 98.

Chiococca sp. Arbusto 1 m. Fr. blanco. Ene. VD. SS 3800.

Diodia teres Walter. Hierba $50 \mathrm{~cm}$. Fl. blanca. Oct. SBC. SAY 5075 .

Exostema caribaeum (Jacq.) Roem. et Schult.

Árbol o arbusto 3-5 m. Fl. blanca. Ago. SBC. ANZ 846; ASM 912.

Genipa americana L. Árbol o arbusto 4-5 m. Fl. blanca. Ago. SBC, SBC2. ASM 982; GJ 2171.

Guettarda elliptica Sw. Árbol o arbusto 1-5 m. Fl. blanca, amarilla. May.-jul. SBC, SBCE, VD. GJ 1752; ASM 290, 776; SAY 5247, 5574.

Hamelia patens Jacq. var. glabra Oerst. Arbusto 3 m. FI. anaranjada. Ago. SBC. ASM 947.

Hamelia versicolor A.Gray. Arbusto o hierba $80 \mathrm{~cm}-4 \mathrm{~m}$. Fl. roja. Ago.-sept. SBC, SBC2. ANZ 1109; SAY 4278; ME 7272; SS 5397.

Hintonia latiflora (Sessé et Moc. ex DC.) Bullock. Árbol o arbusto 2-10 m. Fr. verde. Sept-feb. SBCE, SBC. SS 5378, 5567; SAY 4387, 5404; GJ 1785.

Mitracarpus hirtus (L.) DC. Hierba o hierba rastrera $50 \mathrm{~cm}$. Fl. blanca. Oct. SBC. ANZ 509; ASM 110.

Mitracarpus rhadinophyllus (B.L.Rob.) L.O.Williams. Hierba
$20 \mathrm{~cm}$. Fl. blanca. Sept. Sa. SAY 4244.

Psychotria microdon (DC.) Urb. Arbusto 2-3 m. Fl. verde. Oct. SBC, SMS. SAY 4350, 5119.

Randia aculeata L. Arbusto 1-3 m. Fl. blanca. Jun. SBC, SBC2. SAY 5477, 5608; GJ 2367.

Randia cinerea (Fernald) Standl. Árbol o arbusto 2-4 m. Fr. verde. Oct.-feb. SBC. ASM 1247; ANZ 466; SS 5615.

Randia laevigatoides Borhidi. Árbol 3-9 m. Fl. amarilla. Abr. SBCE, SBC. ASM 1490; ANZ 457, 1000, 1184; SS 5467.

Randia malacocarpa Standl. Arbusto 2 m. Fr. verde. Feb. Sa. ASM 1265.

Randia matudae Lorence et Dwyer. Árbol 3 m. Fl. amarilla. May. SBC. ASM 763.

Randia nelsonii Greenm. Árbol o arbusto 1-6 m. Fr. verde. Jun.-sept. SBC. GJ 1803; ANZ 1189; SAY 5532.

Randia oaxacana Standl. Árbol 6 m. Fr. verde. Sept. SBCE. SAY 4448.

Randia thurberi S.Watson. Arbusto 1-3 m. Fr. verde. Jun.-ago. SBC, SBC2. ASM 1048; SAY 5499.

Richardia scabra L. Hierba 20-40 cm. Fl. blanca. Sept.-oct. SBC, SBC2. ANZ 1269; ASM 1100, 1135.

Simira rhodoclada (Standl.) Steyerm. Árbol 6-7 m. Estéril. SMS, SBC. SS 5636; ASM 1212.

Spermacoce tenuior L. Hierba $60 \mathrm{~cm}$. Fl. blanca. Oct. SBC. SAY 5107.

RUTACEAE

Esenbeckia berlandieri Baill. ex Hemsl. Árbol 4-7 m. Botones amarillos. Jul. SBC. SAY 5575, 5670; ANZ 746; SS 5470; ASM 133.

Esenbeckia collina Brandegee. Árbol 3-4 m. Inflorescencias verdes. Dic. SBC. GJ 1925, 1929; ASM 305.

Zanthoxylum arborescens Rose. Árbol 4.5 m. Estéril. Oct. SBC. G) 1823.

Zanthoxylum melanostictum Schltdl. et Cham.

Árbol 6 m. Fr. verde. Oct. SBC. SAY 5250.

\section{SAPINDACEAE}

Cardiospermum grandiflorum Sw. Bejuco. Fl. blanca. Oct.nov. SBC. ANZ 478; ASM 365.

Cardiospermum halicacabum L. Bejuco. Fl. blanca y amariIla. May.-dic. SBC, SBC2, Sa, SBCE. ANZ 1052, 1093; AÁH 63; SAY 4245, 4454. 
Cardiospermum sp. Bejuco. Fl. blanca. Oct. SBC. ASM 178.

Paullinia costaricensis Radlk. Bejuco. Fr. rojo. Mar. SBC. ASM 725.

Paullinia cururu L. Bejuco. Fr. rojo. Ago.-sept. SBC, SMS. ANZ 1127; ASM 1606; AÁH 89; SAY 4351.

Paullinia fuscescens Kunth. Bejuco. Fl. blanca. May. SBC. SS 5903; ANZ 1362; ASM 1366.

Sapindus saponaria L. Árbol 7 m. Fl. blanca. Feb. SBC. SAY 5343.

Serjania racemosa Schumach. Bejuco. Fl. blanca. Dic. SBC. ASM 515.

Serjania schiedeana Schltdl. Bejuco. Fr. verde. Mar. SBC. ASM 1326.

Serjania triquetra Radlk. Bejuco. Fr. amarillo verdoso. Nov. SBC. ANZ 1287.

Serjania sp. Bejuco. Fl. blanca y estambres amarillos. Oct. SBC. ASM 116.

Thouinia villosa DC. Árbol o arbusto 3-6 m. Fl. crema o verde. May.-jun. SBC, SBC2. GJ 1774, 1784, 2233; SAY 5501; ASM 1479.

Thouinidium decandrum (Bonpl.) Radlk. Árbol 3-10 m. Fl. blanca. Nov.-mar. SBC, SBC2. SAY 5182, 5478; ANZ 932, 1329; ASM 447.

Urvillea ulmacea Kunth. Bejuco. Fl. amarilla. Dic.-ene. SBC, Ga. ANZ 718, 826; ASM 619, 673; SS 5519.

\section{SAPOTACEAE}

Pouteria campechiana (Kunth) Baehni. Árbol 2-13 m. Fl. verde. Feb. SBC. ASM 642, 1236.

Sideroxylon capiri (A.DC.) Pittier. Árbol 8-15 m. Fr. verde feb.-jun. SBC2, SBC. ANZ 1509; ASM 1243.

Sideroxylon celastrinum (Kunth) T.D.Penn. Árbol o arbusto 1.5-10 m. FI. amarilla. Ene.-mar. VD, SBCE, SBC,SBC2. SAY 5376; SS 3798, 5635; ASM 780, 1274.

Sideroxylon lycioides L. Árbol 4.5 m. Estéril. Dic. SBC. G) 1905.

\section{SCROPHULARIACEAE}

Capraria biflora L. Hierba $40 \mathrm{~cm}-1.2 \mathrm{~m}$. Fl. blanca o verde. Jun.-feb. SBC, SBC2, Ga, SBce2, SMS. AÁH 83; ASM 609, 1109; ANZ 676, 1160.

Capraria frutescens (Mill.) Britton. Hierba $50 \mathrm{~cm}$. Fl blanca con puntos lilas. Nov. SBC. ASM 1948.
Schistophragma pusillum Benth. Hierba $15-40$ cm. Fl. morada. Sept. SBC2. ANZ 1232, 1246 .

Scoparia dulcis L. Hierba 70 cm. Fl. blanca. SBC2. AÁH 81.

SIMAROUBACEAE

Recchia connaroides (Loes. et Soler) Standl. Arbusto $6 \mathrm{~m}$. Estéril, corteza escarapelada roja. SBC. G) 1760.

Recchia mexicana Moc. et Sessé ex DC. Árbol 2-7 m. Fl. amarilla. Feb. SBC, SMS, VD. SS 5529, 5638; GJ 1868; ASM 1245, 1293.

\section{SOLANACEAE}

Capsicum annuum L. var. no determinada. Arbusto $40 \mathrm{~cm}-$ $2 \mathrm{~m}$. Fl. amarilla tenue o blanca. Jul.-ago. SBC. ASM 247, 458, 983; SAY 5601, 5641.

Capsicum annuum L. var. glabriusculum (Dunal) Heiser et Pickersgill. Arbusto 1.5 m. Fr. verde o negro. Oct. SBC. ASM 230.

Capsicum rhomboideum (Dunal) Kuntze. Arbusto $1.2 \mathrm{~m}$. Fl. blanca. Jul. SBC. GJ 2678.

Datura discolor Bernh. Hierba $60 \mathrm{~cm}$. FI. blanca. Sept. SMS. SAY 4206.

Datura inoxia Mill. Hierba $40 \mathrm{~cm}$. Fl. blanca. Ago. SBC. ME 5141.

Physalis sp. Hierba $20 \mathrm{~cm}-2 \mathrm{~m}$. Fl. blanca, amarilla o amariIla con el centro morado. Ago.-sept. SBC. ASM 678, 914, 946.

Solanum aculeatum St.-Lag. Bejuco. Fl. blanca verdosa. Sept. SBC2. ANZ 1169.

Solanum adscendens Sendtn. Hierba 20 cm. Fl. blanca. Ago. SBC2. ANZ 1104, 1481, 1504.

Solanum americanum Mill. Arbusto $1.5 \mathrm{~m}$. Fl. blanca. Sept. Ga. SAY 4425.

Solanum campechiense L. Hierba $30 \mathrm{~cm}$. Fl. morada. Abr. SBC. ME 6479; ASM 1343.

Solanum glaucescens Zucc. Bejuco. Fl. verde o amarilla. Oct. SBC. SAY 5197; ASM 243, 349, 1142.

Solanum hazenii Britton. Árbol 2-5 m. Fl. blanca. Oct.-nov. SBC, SBC2. SAY 5039, 5199; ANZ 722, 1299; ASM 484.

Solanum lycopersicum L. var. cerasiforme (Dunal) D.M. Spooner, G.J.Anderson et R.K.Jansen. Hierba $40 \mathrm{~cm}$. Fl. amarilla. Abr. SBC. ASM 1434.

Solanum refractum Hook. et Arn. Bejuco. Fl. amarilla. Jun. SBC2. ANZ 1507. 
Solanum wendlandii Hook.f. Bejuco. Fr. verde. Nov. SBC. ANZ 1308.

\section{STERCULIACEAE}

Ayenia micrantha Standl. Arbusto 2 m. Fr. verde. Sept. SBC. ANZ 1163.

Byttneria aculeata (Jacq.) Jacq. Arbusto decumbente $60 \mathrm{~cm}-2$ m. Fl. morada o guinda. Ago.-nov. SBC, SMS. ASM 149, 194; ANZ 1077; SAY 4327, 5050.

Guazuma ulmifolia Lam. Árbol o arbusto 2-8 m. Fl. amarilla. Abr.-oct. SBC, SBC2. AÁH 85; KV 719; SAY 5469; ASM 632; SS 5884 .

Melochia nodiflora Sw. Arbusto $30 \mathrm{~cm}-1.5 \mathrm{~m}$. Fl. rosa, rojas o salmón sept.-abr. SBC, SBC2. ANZ 535, 964; ASM 218, 336; SS 5505.

Melochia tomentosa L. Arbusto $30 \mathrm{~cm}-1.5 \mathrm{~m}$. Fl. púrpura o rosa intenso o blanca con morado. Ago.-dic. SBC, SBC2, SBce2. ANZ 470, 1158; ASM 155, 409; SS 5511.

Waltheria indica L. Hierba sufrutescente $30 \mathrm{~cm}-1.5 \mathrm{~m}$. FI. amarilla, roja, amarilla con lila o amarilla con rojo. Sept.may. SBC, SBC2. ANZ 528, 697; ASM 210, 386; KV 709.

\section{THEOPHRASTACEAE}

Jacquinia aurantiaca W.T.Aiton. Arbusto 2-3 m. Fl. roja. Feb. SBC. ANZ 970; SAY 5287.

Jacquinia donnell-smithii Mez. Árbol o arbusto 1-1.7 m. FI anaranjada, roja o amarilla. Dic.-may. SBCE, SBC, SBC2, Ma. SS 5568, 5624; ASM 140, 507; SAY 5401.

Jacquinia macrocarpa Cav. Árbol o arbusto 1-5 m. Fl. rojas o anaranjadas. Nov.-jun. SBC, SBC2. GJ 1751, 1900; ASM 157, 490; ANZ 440.

Jacquinia pungens Gray. Arbusto $3 \mathrm{~m}$. Botones. Ene. SBC. ASM 591.

\section{TILIACEAE}

Corchorus aestuans L. Hierba $80 \mathrm{~cm}$. Fl. amarilla. Sept. SBC2. ANZ 1229.

Heliocarpus donnellsmithii Rose. Árbol 4-6 m. Fl. amarilla verdosa. Dic.-ene. SBC. ANZ 815; ASM 342, 459; SS 5476; G) 1842 .

Heliocarpus occidentalis Rose. Árbol 3 m. Fl. blanca. Feb. SBC. SS 5545.

Heliocarpus pallidus Rose. Árbol 5 m. Botones. Nov. SBC. ASM 426.

Luehea candida (Moc. et Seseé ex DC.) Mart. Árbol o arbusto 3-6 m. Fl. blanquecina. Jul. SBC, SMS. ANZ 818, 1122; SAY 4215, 4409; GJ 1799.
Triumfetta falcifera Rose. Arbusto $2 \mathrm{~m}$. Fl amarilla. Nov. SBC. ASM 1947.

Triumfetta semitriloba Jacq. Árbol 4-8 m. Fl. verde o amarilla. Oct.-ene. SBC. ANZ 460, 539, 698; ASM 338.

\section{TRIGONIACEAE}

Trigonia eriosperma (Lam.) Fromm et Santos Bejuco. Fl. café. Feb. SBC. ANZ 965.

\section{TURNERACEAE}

Turnera diffusa Willd. ex Schult. Arbusto $30 \mathrm{~cm}-1 \mathrm{~m}$. Fl. amarilla. Todo el año. Sa, SBC, SBC2. SAY 4234, 4238a; ASM 508, 968; GJ 2179.

\section{ULMACEAE}

Celtis caudata Planch. Árbol o arbusto 2-4 m. Fr. verde. Jun.oct. SBC, SBC2. ASM 70, 1594.

Celtis iguanaea (Jacq.) Sarg. Árbol o arbusto 3-7 m. Fr. verde o amarillo. Jun.-nov. SBC, SBC2, SMS. SAY 4296, 4374, 5111; ANZ 1501; ASM 374, 996.

Phyllostylon rhamnoides (J.Poiss.) Taub. Árbol 80 cm-2.5 m. Fl. rosa mexicano. Oct. SBC. ASM 1322, 1944.

Trema micrantha (L.) Blume. Árbol 4-6 m. Fr. verde. Jul.-oct. SBC. SAY 5084, 5716.

\section{URTICACEAE}

Pouzolzia guatemalana (Blume) Wedd. var. nivea (S.Watson) Friis et Wilmot-Dear. Árbol 4 m. Fl. verde. Oct. SBC. SAY 5193.

Urera pacifica V.W.Steinm. Árbol 4 m. Fl. blanca. Jun. SBC2. ANZ 1494; SAY 5628.

\section{VERBENACEAE}

Avicennia germinans (L.) L. Árbol $4 \mathrm{~m}$. Fl. amarilla. Sept. SBC2. ANZ 1221.

Bouchea nelsonii Grenzeb. Hierba 40-70 cm. Fl. lila pálido a morada. Oct. SBC, SBC2. ASM 15, 82; ANZ 510.

Lantana camara L. Arbusto $40 \mathrm{~cm}-2 \mathrm{~m}$. Fl. de centro amarillo y periferia roja. Jul.-dic. SBC, SBC2. ASM 31, 76; SAY 4276, 5023; SS 5464.

Lippia umbellata Cav. Árbol 3.5-7.5 m. Fl. amarilla-verdosa. Oct. SBC. GJ 1749, 1789.

Phyla nodiflora (L.) Greene. Hierba o hierba rastrera 20-50 cm. Fl. blanca. Abr.-sept. SBC, SBC2, Ga, SMS. AÁH 52; ANZ 1243, 1372; ASM 441; SAY 4368.

Priva lappulacea (L.) Pers. Hierba $30-80 \mathrm{~cm}$. Fl. blanca. Ago. SBC, SBC2. SAY 5606, 5756; ASM 981, 1609; ANZ 578.

Stachytarpheta frantzii Pol. Hierba $40-60 \mathrm{~cm}$. Fl. azul a morada. Jun.-sept. SMS, SBC, SBC2. SAY 4202; ANZ 1277b, 1484; ASM 979. 
Vitex hemsleyi Briq. Árbol 4-12 m. FI. morada. May. SBC, SBC2. SAY 4414; KV 745; GJ 2192; ASM 1614.

Vitex mollis Kunth. Árbol o arbusto $80 \mathrm{~cm}-7 \mathrm{~m}$. FI. lila. SAY 4303, 4391; SS 3801, 5556; ASM 712.

\section{VIOLACEAE}

Hybanthus attenuatus (Humb. et Bonpl. ex Roem. et Schult.) Schulze-Menz. Hierba 50-80 cm. Fr. verde. Oct.-dic. SBC. SAY 5103, 5112; ANZ 678, 679; ASM 487.

Hybanthus mexicanus Ging. Árbol o arbusto 2.5-3 m. Fr. verde. Oct. SBC. SAY 5131, 5165, 5219; GJ 1810; ASM 238.

\section{VITACEAE}

Ampelocissus acapulcensis (Kunth) Planch. Bejuco. Fl. roja. Mar. SBC, SBC2. GJ 2049; SAY 5468.

Ampelopsis mexicana Rose. Arbusto escandente o bejuco 3 m. Fl. rojo-verdosa. Feb. SBC, SBC2. ANZ 1355; G) 1936, 1952, 2039; ASM 792.

Cissus alata Jacq. Bejuco. Fr. verde o negro. Nov. SBC. GJ 1859.

Cissus sicyoides L. Bejuco. Fl. amarilla o verde. Sept.-abr. SBC, SBC2, Sa, SBCE2. ANZ 1156, 1261; SAY 4220, 5125; ASM 930.

Vitis sp. Bejuco. Fl. rosa. Sept. SBC. SAY 4323.

\section{ZYGOPHYLLACEAE}

Guaiacum coulteri A.Gray. Árbol o arbusto postrado $60 \mathrm{~cm}$ $8 \mathrm{~m}$. Fl. lila o morada. Mar.-oct. SBC, SBC2, SBCE, SBce2, VD. ASM 180, 637; SAY 4285, 4452; SS 5481.

Kallstroemia brachystylis Vail. Hierba rastrera. FI. blanca. Jul.-ago. SBC. ASM 969; SAY 5725.

\section{LILIOPSIDA}

\section{AGAVACEAE}

Agave angustifolia Haw. Arbusto $90 \mathrm{~cm}-1 \mathrm{~m}$. Fl verde. Oct. SBC, VD. SS 3806; ASM 45.

\section{ARACEAE}

Philodendron hederaceum (Jacq.) Schott. Bejuco. FI inmadura. Nov. SBC. GJ 1856.

Pistia stratiotes L. Hierba acuática. Fl blanca. Abr. Laguna. ANZ 1369; SAY 4379.

\section{BROMELIACEAE}

Aechmea sp. Epífita herbácea. SBC. ANZ 1385.

Bromelia karatas L. Arbusto $80 \mathrm{~cm}$. Hojas en roseta. Fr verde en el centro de la roseta. Oct. SBC. SAY 5181.

Bromelia palmeri Mez. Hierba $1 \mathrm{~m}$. Fl amarilla. Jul. SBC. ASM 166; SAY 5597.
Bromelia pinguin L. Hierba rosetófila $40 \mathrm{~cm}$. Fr amarillos o verdes. Mar.-jul. SBC, SBCE. GJ 2043, 2688.

Tillandsia caput-medusae E.Morren. Epífita herbácea. VD, Ma. SAY 5380.

Tillandsia maritima Matuda. Epífita herbácea $30 \mathrm{~cm}$. Fr verde. Ago. SBC. ANZ 1088b.

Tillandsia polystachia (L.) L. Epífita herbácea $60 \mathrm{~cm}$. SBC. ASM 1289

Tillandsia sp. Epífita herbácea. SBC. SAY 5564.

\section{CANNACEAE}

Canna sp. Hierba 70 cm. Fr verde. Ene. SBC. ANZ 885.

\section{COMMELINACEAE}

Commelina diffusa Burm.f. Hierba $30-50 \mathrm{~cm}$. FI blanca o rosa. Sept.-oct. SBC. ASM 112; SAY 4318.

Commelina erecta L. Hierba 40-60 cm. Fl blanca. Ago.-oct. SBC. AÁH 80; ANZ 435; ASM 67; SAY 5224.

Tinantia sp. Hierba 10-50 cm. Fr verde. Oct. SBC. SAY 5042, 5207.

\section{CYPERACEAE}

Cyperus canus J.Presl et C.Presl. Hierba $60 \mathrm{~cm}-1 \mathrm{~m}$. Fl blanca o verde. Ene.-feb. SBC, Ga. ANZ 663; ASM 585, 599; SAY 5122, 5312.

Cyperus chorisanthos C.B.Clarke. Hierba. Fl café. Ago. SBC. AÁH 86.

Cyperus hermaphroditus (Jacq.) Standl. Hierba 35 cm-1 m. Fr verde. Sept.-oct. SBC, G. SAY 4431, 5044.

Cyperus odoratus L. Hierba $80 \mathrm{~cm}$. Fr verde. Sept. Ga. SAY 4430.

Cyperus sp. Hierba 25 cm. Fr verde. Oct. SBC. SAY 5014.

\section{DIOSCOREACEAE}

Dioscorea convolvulacea Schltdl. et Cham. Bejuco. Fl blancas. Oct. SBC. ASM 1192.

Dioscorea nelsonii Uline ex R.Knuth. Bejuco. Fr verde. Ago. SBC. ASM 1020.

Dioscorea preslii Steud. Bejuco. FI verde. Oct. SBC. ASM 102.

Dioscorea sp. Bejuco. FI verde. Oct. SBC. SAY 5120.

LILIACEAE

Hymenocallis howardii Bauml. Hierba $50 \mathrm{~cm}$. Fl. blanca. May. SBC. SS 5931b. 


\section{MARANTACEAE}

Maranta arundinacea L. Hierba $30 \mathrm{~cm}-1 \mathrm{~m}$. Fl. blanca. Jul.oct. SBC, SBC2. ASM 22, 353; ANZ 1049; SAY 4214, 5132.

\section{ORCHIDACEAE}

Barkeria schoemakeri Halb. Epífita herbácea $15 \mathrm{~cm}$. Fl. rosa y labelo rosa mexicano. Feb. SBC. ASM 1279.

Clowesia dodsoniana Ag.León. Epífita herbácea. Fl. verde con líneas verdes. May. SBC, SBC2. ANZ 1171; GJ 1963, 2199; ASM 1312.

Encyclia adenocarpon (La Llave et Lex.) Schltr. Epífita herbácea $40 \mathrm{~cm}$. Fr. verde. Ene. SBC. KV 700.

Encyclia rzedowskiana Soto Arenas. Epífita herbácea. Fl. verde. Feb. SBC. ASM 1281.

Erycina echinata (Kunth) Lindl. Epífita herbácea. Estéril. SBC. SS 5920; ASM 1280.

Trichocentrum cebolleta (Jacq.) M.W.Chase et N.H.Williams. Epífita herbácea 35-40 cm. Fl. amarilla moteada de café. Ene.-feb. SBC, SBC2, Sa. ASM 1267, 1278; GJ 2172; KV 694.

\section{POACEAE}

‡ Arundo donax L. Hierba $1.2 \mathrm{~m}$. Inflorescencia beige. Jul. SBCE. GJ 2696.

Bouteloua chondrosioides (Kunth) Benth. ex S.Watson. Hierba 25 cm. Fr. verde. Ago. SBC. ASM 975.
Cenchrus brownii Roem. et Schult. Hierba 80 cm. Fl. verde. Jul. SBC. GJ 2671.

‡ Cenchrus ciliaris L. Hierba $40 \mathrm{~cm}$. Fr. verde y café. Dic. SBC. ANZ 675.

Chloris barbata Sw. Hierba 60 cm. Fr. verde o café. Jul.-dic. SBC. ANZ 689; SAY 5623a.

‡ Dactyloctenium aegyptium (L.) Willd. Hierba 5-70 cm. Fr. verde. Jul.-feb. SBC, SBC2, SMS. ASM 303; SAY 4194, 5345, 5711; ANZ 1245.

‡ Echinochloa colona (L.) Link. Hierba 50-60 cm. Fr. verde. Sept.-ene. SBC, SBC2. ANZ 896, 1274.

Eragrostis ciliaris (L.) R.Br. Hierba $60 \mathrm{~cm}$. Fr. verde y café. Dic. SBC. ANZ 680.

Jouvea pilosa (J.Presl) Scribn. Hierba 20 cm. Fr. café. Feb. VD. SAY 5382.

‡ Melinis repens (Willd.) Zizka. Hierba $30-80 \mathrm{~cm}$. FI. roja o rosa. Sept.-oct. SMS, SBC, SBC2. SAY 4198, 5081; ASM 267, 665; ANZ 1271.

Panicum muticum Forssk. Hierba 1.50 m. Fr. verde. Jul. SBC. ME 6977.

Setaria liebmannii E.Fourn. Hierba $50-70 \mathrm{~cm}$. Fl. verde con tonos morados. Jul. SBC. GJ 2669; ASM 1627. 